\title{
The revised GB/GB2 sample of extragalactic radio sources $^{\star}$
}

\author{
J. Machalski ${ }^{1}$ \\ Obserwatorium Astronomiczne, Uniwersytet Jagielloński, ul. Orla 171, 30-244 Kraków, Poland
}

Received April 16; accepted June 25, 1997

\begin{abstract}
This paper presents the revised sample of 373 extragalactic radio sources brighter than $0.2 \mathrm{Jy}$ at 1.4 GHz. These sources, selected from the finding Green Bank surveys, were mapped at $1465 \mathrm{MHz}$ using the VLA at different configurations. The biases introduced into the original GB and GB2 catalogues by confusion as well as partial resolution by the VLA at A-configuration, are eliminated. In effect, a number of sources have been excluded, and a few other are included into the revised sample. Now the sample is about 99,97 , and 95 per cent complete for sources with $S_{1.4} \geq 0.55 \mathrm{Jy}, 0.25 \mathrm{Jy} \leq S_{1.4}<0.55 \mathrm{Jy}$, and $0.2 \mathrm{Jy} \leq S_{1.4}<0.25 \mathrm{Jy}$, respectively. A compilation of the radio, optical, and X-ray data available for the sample sources are presented in Table 3. New 4.9-GHz VLA images of selected sources are included. A number of statistics describing radio morphological and spectral contents of the sample, radio variability, revised source counts, redshift distributions, etc. are given.
\end{abstract}

Key words: surveys - quasars: general - galaxies: active - radio continuum: general; galaxies

\section{Introduction}

Statistically complete samples of extragalactic radio sources, providing as much as possible information about their physical and geometrical parameters, radio structure and morphology, optical and X-ray counterparts, etc., are still of great importance for further astrophysical and cosmological studies. The best studied samples, complete within a defined sky area and flux-limited only, were selected from finding surveys of the sky carried out at a num-

Send offprint requests to: J. Machalski

e-mail: machalsk@oa.uj.edu.pl

* Table 3 and notes to Table 3 are also available in electronic form at CDS via ananymous ftp to cdsarc.u-strasbg.fr (130.79.128.5) or via http://cdsweb.u-strasbg.fr/Abstract. html ber of frequencies. In particular, much observational attention have been given to the brightest sources at $178 \mathrm{MHz}$ (10-Jy sample: Laing et al. 1983, and references therein), $408 \mathrm{MHz}$ (B3-VLA sample: Vigotti et al. 1989), $2.7 \mathrm{GHz}$ (2-Jy sample: Wall \& Peacock 1985; cf. also Morganti et al. 1993; Tadhunter et al. 1993), and 5 GHz (S5 sample: Kühr et al. 1981; 1.3-Jy sample: Pearson \& Readhead 1988; CJ1 0.7-Jy sample: Polatidis et al. 1995).

A similar effort has been undertaken at $1.4 \mathrm{GHz}$. About 240 intermediate-strength radio sources stronger than $0.55 \mathrm{Jy}$ and located within a sky area of $0.44 \mathrm{sr}$ were selected by Machalski \& Maslowski (1982) (hereafter referred to as Paper I) from the GB and GB2 finding surveys, and afterwards supplemented with those having $S \geq 0.2$ Jy and $S \geq 0.25 \mathrm{Jy}$, respectively, in two smaller regions corresponding the area of $0.09 \mathrm{sr}$ (Machalski \& Condon 1983b). The sample sources were then observed with the VLA at $1465 \mathrm{MHz}$ or $1490 \mathrm{MHz}$ in its partial (P) or A-configuration. High-resolution $(1.3-2.5$ arcsec $)$ maps were provided for all the sources (Machalski et al. 1982; Paper II, Machalski \& Condon 1983a,b; Papers III and IV, respectively). Strongly resolved and/or confused sources were reobserved with the VLA C-array, providing low-resolution maps (Machalski \& Condon 1985a; Paper V). As a result, the radio structure, angular size, asymmetry, bending, etc. could be determined for the extended sources. As expected, the sample consists of a large fraction of compact sources, unresolved with the VLA synthesized beam of about $1.2-1.3$ arcsec; those were analysed by Machalski \& Inoue (1990).

The described VLA observations showed that several GB and GB2 radio sources were strongly confused by a fainter companion closer than about $6-7$ arcmin, or even appeared to be a cluster of a few nearby sources unresolved with the $\approx 10-11$ arcmin beam of the NRAO 91 $\mathrm{m}$ telescope used in the finding surveys. Furthermore, the information content of the sample concerning optical identification, photometry, redshift, X-ray counterpart, etc., has grown since the above publications due to our own observational efforts, as well as results published by other 
authors investigating objects common with the GB/GB2 sample.

The present paper concludes the sample revising its completeness to the limiting flux of $0.2 \mathrm{Jy}$, and providing a compendium of the radio, optical, and X-ray parameters for the sample sources. In Sect. 2 a definition of the sample is given, and its completeness is estimated. Designation of the radio spectrum, morphology, and variability is given in Sect. 3, while the optical identification with galaxies and quasars, their magnitudes and redshift, are verified in Sect. 4 . The radio, optical, and recent X-ray data for the sample sources are summarized in Sect. 5. Some statistical results: radio morphology and optical identification contents of the sample, revised 1.4-GHz source counts around their peak at about $0.6 \mathrm{Jy}$, precise spectral-index distributions, and redshift distributions, are described in Sect. 6 .

\section{The sample}

\subsection{Definition of the sample}

The sample is defined as follows:

Part 1: 233 sources with $S_{1.4} \geq 0.55$ Jy (hereafter on the Baars et al. 1977 scale) within the $\mathrm{B}(1950)$ sky coordinates: $7^{\mathrm{h}} 17^{\mathrm{m}}<\alpha<16^{\mathrm{h}} 23^{\mathrm{m}},+45^{\circ} 8<\delta<+51^{\circ} 7$ and $7^{\mathrm{h}} 08^{\mathrm{m}}<\alpha<16^{\mathrm{h}} 58^{\mathrm{m}},+31^{\circ} 8<\delta<+39^{\circ} 7$. Sky area of $0.4414 \mathrm{sr}$.

Part 2: 109 sources with $0.25 \mathrm{Jy} \leq S_{1.4}<0.55 \mathrm{Jy}$ within $7^{\mathrm{h}} 15^{\mathrm{m}}<\alpha<13^{\mathrm{h}} 11^{\mathrm{m}},+48^{\circ} 0<\delta<+50^{\circ} 0$, $7^{\mathrm{h}} 08^{\mathrm{m}}<\alpha<9^{\mathrm{h}} 08^{\mathrm{m}},+34^{\circ} 5<\delta<+38^{\circ} 0$, and $9^{\mathrm{h}} 08^{\mathrm{m}} \leq \alpha<16^{\mathrm{h}} 57^{\mathrm{m}},+34^{\circ} 5<\delta<+35^{\circ} 5$. Sky area of $0.0906 \mathrm{sr}$.

Part 3: 31 sources with $0.20 \mathrm{Jy} \leq S_{1.4}<0.25 \mathrm{Jy}$ within $7^{\mathrm{h}} 08^{\mathrm{m}}<\alpha<9^{\mathrm{h}} 08^{\mathrm{m}},+34^{\circ} 5<\delta<+38^{\circ} 0$, and $9^{\mathrm{h}} 08^{\mathrm{m}} \leq \alpha<16^{\mathrm{h}} 57^{\mathrm{m}},+34^{\circ} 5<\delta<+35^{\circ} 5$. Sky area of $0.0550 \mathrm{sr}$.

All the sources have $b_{\mathrm{II}}>20^{\circ}$. Hereafter these parts are referred to as Subsample 1, 2, and 3, respectively.

\subsection{Mean weighted 1.4-GHz flux density}

For all the sample sources their two independent measurements of $1.4 \mathrm{GHz}$ flux density resulting from the Green Bank surveys GB/GB2 (Maslowski 1972; Machalski 1978a) and 83GB (Condon \& Broderick 1985; the relevant catalogue by White \& Becker 1992) were compared with the measured VLA flux density (cf. Introduction). That comparison allowed to select either confused sources requiring flux correction or heavily resolved sources for further reobservations with lower resolution. Some of the confused sources appeared to be two different ones but still satisfying the criteria of the sample. Recently D-array and B-array maps for several of the sample sources have become available from the large VLA 1.4-GHz sky surveys: FIRST by Becker et al. (1995) and Condon et al. (1996). These were also used to check the effects of resolution in our data.
For many of the sources, additional $1.4 \mathrm{GHz}$ fluxes were also available from observations with the Effelsberg $100 \mathrm{~m}$ telescope (Kühr et al. 1981) and Arecibo $300 \mathrm{~m}$ telescope (Owen et al. 1983); some fluxes for compact sources were available from measurements with the WSRT and Cambridge One-mile (OMT) telescopes. All collected $1.4 \mathrm{GHz}$ flux densities, corrected for confusion if necessary, were then used to compute the weighted mean flux density at this frequency, $\left\langle S_{1.4}\right\rangle$, and its error, $\sigma$, with the standard formulae

$$
\sigma^{2}=\left[\sum_{i}\left(1 / \sigma_{i}^{2}\right)\right]^{-1} \quad\left\langle S_{1.4}\right\rangle=\sum_{i}\left(S_{i} \sigma^{2} / \sigma_{i}^{2}\right) .
$$

The mean 1.4-GHz flux density was used to verify completeness of the sample.

\subsection{Completeness of the sample}

In order to satisfy the conditions given in Sect. 2.1, 12 sources from Paper I and 20 sources observed in Papers IV and V have been excluded from the sample. They are listed in Table 1 with information about the cause of exclusion. In turn, 15 sources have been added to the sample because they were underestimated in the finding surveys due to a large flux density error but their mean 1.4$\mathrm{GHz}$ flux density calculated from all available observations (cf. Sect. 2.2) satisfied the selection criterion. These additional sources are indicated by a diamond mark $(\diamond)$ preceding the source name in Table 3 . Due to the above corrections, we estimate that now the sample is about 99 per cent complete for sources with $S_{1.4} \geq 0.55 \mathrm{Jy}$, about 97 per cent complete for sources with $0.25 \mathrm{Jy} \leq S_{1.4}<$ $0.55 \mathrm{Jy}$, and about 95 per cent complete for sources with $0.20 \mathrm{Jy} \leq S_{1.4}<0.25 \mathrm{Jy}$. The completeness is evaluated using the method of Dixon \& Kraus (1968).

\section{The radio data}

\subsection{Radio spectrum and spectral index}

In order to determine the radio spectrum for each source of the sample, we relied on a number of radio source catalogues, especially on: $151-\mathrm{MHz}$ 6CII, III, and IV (Hales et al. 1993; and references therein, $365-\mathrm{MHz}$ Texas (Douglas et al. 1980), 408- $\mathrm{MHz}$ B2 and B3 (Colla et al. 1973 and Ficarra et al. 1985, respectively), 1.4- $\mathrm{GHz}$ Arecibo observations in the sky strip $+34^{\circ} 5<$ $\delta<+35^{\circ} 5$ (Owen et al. 1983) and 83GB (White \& Becker 1992), 4.8-GHz GB6 (Gregory et al. 1996) and MGII and MGIV (Langston et al. 1990 and Griffith et al. 1991, respectively). The flux densities were adjusted to the common scale of Baars et al. (1977). Errors of the flux density were taken directly from the individual papers or calculated according to the formulae given there. A fitting the data points with a polynomial function of the type 
Table 1. (left panel): Sources excluded from the Part 1 (list of Machalski \& Maslowski 1982); (central and right panel): excluded from the Part 2 and Part 3 (lists of Machalski \& Condon 1983b, 1985)

$\begin{array}{lllllll}\text { Source } & \text { Cause } & \text { Source } & \text { Cause } & \text { Source } & \text { Cause } & \text { Note } \\ & & & & & & \\ 0724+467 & S<0.55 \mathrm{Jy} & 0719+362 & 2 \text { sources } & 1137+493 & S<0.25 \mathrm{Jy} & \\ 0738+336 & 2 \text { sources } & 0728+364 & 2 \text { sources } & 1202+488 & \text { cluster } & \\ 0908+340 & 2 \text { sources } & 0846+377 & 2 \text { sources } & 1204+483 & 2 \text { sources } & \\ 0913+471 & S<0.55 \mathrm{Jy} & 0851+363 & 2 \text { sources } & 1205+500 & 2 \text { sources } \\ 0914+502 & S<0.55 \mathrm{Jy} & 0902+480 & 2 \text { sources } & 1225+498 & 2 \text { sources } & \\ 1112+333 & 2 \text { sources } & 0910+486 & S<0.25 \mathrm{Jy} & 1316+346 & S<0.20 \mathrm{Jy} & \\ 1135+464 & 2 \text { sources } & 0954+490 & 2 \text { sources } & 1401+350 & \text { cluster } & \text { a) } \\ 1317+362 & 2 \text { sources } & 0955+492 & 3 \text { sources } & 1533+345 & \text { cluster } & \\ 1432+382 & 3 \text { sources } & 1042+481 & S<0.25 \mathrm{Jy} & 1539+350 & S<0.20 \mathrm{Jy} & \\ 1442+363 & 2 \text { sources } & 1124+488 & \text { cluster } & 1648+350 & 2 \text { sources } & \\ 1614+473 & S<0.55 \mathrm{Jy} & & & & & \end{array}$

a) given in Machalski \& Condon (1990).

$y=a+b x+c x^{2}+d \cdot \exp ( \pm x)$, where $y=\log S, x=\log \nu$, was performed.

Three cases are considered: (1) a straight line, where $c=d=0$ (s-spectrum), (2) a curved fit, where $c=0$ or $d=0$ (c- or c + spectrum), and (3) a composite fit, where $b, c, d \neq 0(+\mathrm{c}-$ spectrum if $b>0$ and $d<0$, or $-\mathrm{c}+$ spectrum if $b<0$ and $d>0)$. The reduced $\chi^{2}$-test was used to decide whether the data could be fitted with either of the above functions. The spectra which could not be adequately fitted to the data (typical for variable sources) are considered to be complex (cpx-spectrum). In some sources the best fitted spectrum consists of two straight lines with a break frequency (sb-spectrum).

The fitted spectrum was then used to determine a spectral index as a derivative (i.e. a slope) of the above type function at the frequency of $1.4 \mathrm{GHz}, \alpha_{1.4}$. Thus, using a functional form of the spectrum, one has two advantages in respect to the popular two-point spectral indices: (i) the slope is not affected by the errors of individual flux density measurements, and (ii) it can be easily transformed into another frequency, e.g. emitted one. In Sect. 6, statistics of the spectra and distributions of $\alpha_{1.4}$ are given and discussed.

\subsection{Radio morphology}

The radio morphology is determined mostly on the basis of VLA maps published in Papers II, III, IV, and V. Because the strongest sample sources (mostly 3C sources with $S_{1.4}>2 \mathrm{Jy}$ ), as well as strong compact sources which had been already observed with the VLA by Perley (1982), were not reobserved during the GB/GB2 project, their morphology was specified from other publications. Extended double sources are classified either as edgebrightened (FRII) or edge-darkened (FRI)(classification of Fanaroff \& Riley 1974), although morphological type of some of the sources should be determined as an intermediate type.

The other sources have either compact structure dominated by a flat-spectrum unresolved radio core, frequently with weak one-sided $(\mathrm{C}+1 \mathrm{~s})$ or two-sided $(\mathrm{C}+2 \mathrm{~s})$ extended emission detected with the VLA, or compact steepspectrum (CSS) structure. In some cases a distinction between these two types is not easy. A compact source with well fitted spectrum of "s" or "c-" type is classified as CSS regardless of a frequency of the maximum of its fitted spectrum, while a compact core-dominated source with detected or undetected " $1 \mathrm{~s}$ " or " $2 \mathrm{~s}$ " emission, having more complex spectrum of "cpx", "+c-" or "-c+" type is considered here as a separate morphological category. It was shown by Machalski \& Inoue (1990) that the GB/GB2 sources with deconvolved angular size $\theta<1-1.5$ arcsec differ significantly in the fringe visibility function, suggesting a variety of structures on the angular scale of about $0.1-0.5$ arcsec, and have radio spectra which can be only conventionally classified as flat or steep. The distribution of $\alpha_{1.4}$ for the CSS sources (Fig. 1) illustrate the problem.

The morphological types and structural parameters of the sources added to the sample have been taken from available maps of the NVSS and FIRST surveys, or from our own, unpublished, VLA maps. The observed structures were crudely deconvolved into elliptical Gaussian components; relevant data, i.e. their map coordinates, integrated $1.4 \mathrm{GHz}$ flux density, half-intensity major and minor diameters, major-axis position angle (degrees east of north), as well as the source's "largest angular size" $L A S$ and "overall position angle" $O P A$, are given in the Appendix (Table A1). 


\section{3. $1.4-G H z$ radio variability}

Due to the large time base of the $1.4-\mathrm{GHz}$ observations (Sect. 2.2), from 8 to 13 years, and a sufficient number of independent flux measurements, it was possible to determine the sample sources which vary significantly at this frequency. The $1.4-\mathrm{GHz}$ variability in the GB/GB2 sample was the subject of separate analyses (Ryś \& Machalski 1990; Machalski \& Magdziarz 1993a). After supplementing older data in the sample with those already available from the NVSS and FIRST surveys, the time base over which the $1.4-\mathrm{GHz}$ variability has been observed extends to 18-24 years. Compactness of these sources and the lack of nearby confusing neighbours (except of $0804+499 \mathrm{~B}$, whose flux densities have been carefully corrected for confusion) provide that their flux densities measured with single-dish telescopes and the VLA are comparable.

The variable sources in the sample are listed in Table 2 . For each source, the variability parameters as defined by Machalski \& Magdziarz (1993a), i.e. the number of independent observations, $n$, mean weighted 1.4-GHz flux density and its error (calculated with Eqs. (1)), chi-squared statistics, "apparent fluctuation" of flux density around its mean value, $Y(n)$ (the $Y(n)$ statistics has the physical meaning of the quantity $\left(S_{\max }-S_{\min }\right) /\left(S_{\max }+S_{\min }\right)$, but is not numerically identical with it), and time lag between the first and last observations, $T$, are given in consecutive columns of Table 2 . Note that all of these variables but one $(1213+350)$ are of complex $(\mathrm{cpx})$ spectrum with $\alpha_{1.4}<0.5$. It is worth to emphasize that most of these sources are known to be variable at frequencies higher than $1.4 \mathrm{GHz}$; those which were or are systematically monitored for variability are marked in the last column of Table 2.

\section{The optical and X-ray data}

\subsection{Optical identifications}

The radio sources were identified with their optical counterpart on the basis of its positional coincidence with the radio centre. If a compact radio core was detected, then it undoubtedly coincided with the identification. If a source was an extended one and no core could be detected, then a much larger area near the "centre" of the source had to be considered. Usually the source was identified with the brightest galaxy or stellar object in the "centre". Majority of the sample sources were identified with galaxies and quasars, as well as galaxy or quasar candidates seen on the Palomar Observatory Sky Survey (POSS) prints. The tentative samples of GB/GB2 radio galaxies and quasar candidates were analyzed by Machalski \& Condon (1985b, 1986). Later on, deeper identifications were collected from the literature and our own research (e.g. Machalski \& Magdziarz 1993b). In Notes to Table 3 some ambiguous identifications are discussed. Unfortunately, still about 34
Table 2. The sample sources variable at $1.4 \mathrm{GHz}$

\begin{tabular}{|c|c|c|c|c|c|c|c|}
\hline Source & $n$ & $\begin{array}{l}\left\langle S_{1.4}\right\rangle \\
{[\mathrm{mJy}]}\end{array}$ & $\begin{array}{c}\sigma \\
{[\mathrm{mJy}]}\end{array}$ & $\chi^{2}$ & $Y(n)$ & $T[\mathrm{yr}]$ & Note \\
\hline $0711+356$ & 7 & 1642 & 12 & 190.0 & 0.08 & 24.1 & \\
\hline $0723+488$ & 4 & 473 & 5 & 87.0 & 0.10 & 24.0 & \\
\hline $0748+333$ & 5 & 684 & 6 & 191.6 & 0.11 & 19.2 & \\
\hline 0804+499B & 5 & 846 & 11 & 180.2 & 0.16 & 24.0 & a) \\
\hline $0828+493$ & 6 & 1123 & 10 & 466.0 & 0.18 & 23.8 & a) \\
\hline $0923+392$ & 9 & 2666 & 32 & 79.7 & 0.07 & 24.2 & a)b)c) \\
\hline $1015+359$ & 5 & 653 & 11 & 71.6 & 0.13 & 24.1 & a) \\
\hline $1128+385$ & 5 & 756 & 8 & 49.1 & 0.07 & 18.3 & a) \\
\hline $1144+352$ & 6 & 555 & 9 & 93.2 & 0.13 & 18.3 & \\
\hline $1155+486$ & 3 & 330 & 15 & 46.9 & 0.37 & 13.0 & \\
\hline $1213+350$ & 6 & 1522 & 20 & 34.6 & 0.06 & 18.6 & a) \\
\hline $1239+376$ & 4 & 569 & 12 & 44.8 & 0.14 & 19.2 & \\
\hline $1308+326$ & 6 & 1405 & 17 & 255.6 & 0.15 & 18.8 & b)c) \\
\hline $1600+335$ & 6 & 2660 & 19 & 32.5 & 0.03 & 23.8 & \\
\hline $1611+343$ & 7 & 2854 & 17 & 391.8 & 0.09 & 23.8 & b) \\
\hline $1633+382$ & 7 & 2112 & 15 & 639.8 & 0.13 & 23.8 & a)b)c) \\
\hline $1656+348$ & 8 & 454 & 6 & 60.3 & 0.08 & 18.4 & a) \\
\hline
\end{tabular}

a) variable at $10.8 \mathrm{GHz}$ (Seielstad et al. 1983)

b) monitored at 4.8, 8.0, and $14.5 \mathrm{GHz}$ (Hughes et al. 1992)

c) monitored at 22.2 and $36.7 \mathrm{GHz}$ (Valtaoja et al. 1992).

per cent of the sources in Part 1, about 45 per cent in Part 2 , and about 52 per cent in Part 3 remain unidentified.

\subsection{Optical magnitudes and redshift}

Red $(R)$ and blue $(B)$ magnitudes of the identified objects are either photometric ones in the $R$ Kron-Cousins $V R I$ system (Cousins 1976) and in the $U B V$ system of Johnson (1966), or estimated from the $\operatorname{POSS}(\mathrm{E})$ and $\operatorname{POSS}(\mathrm{O})$ prints, respectively. In particular, $U B V R I$ photometry for the brightest elliptical galaxies and quasars in the sample was provided by Machalski \& Wiśniewski (1988), and absolute $M_{R}$ and $M_{B}$ magnitudes as well as four intrinsic colours of the elliptical galaxies, corrected for galactic extinction, aperture, and reddening (K-correction), was given by Machalski (1988). Statistics of the optical type is given in Sect. 6 .

Spectroscopic redshift is available for majority of the sample quasars, and for many brighter galaxies. References to the available photometry and redshifts are provided in Table 3. For elliptical galaxies without spectroscopic redshift but with photoelectrical photometry, a redshift estimate, based on corrected $R$-magnitude and $B-R$ colour, is provided by Machalski (1988). A statistical analysis, described in Sect. 6 suggests that at least 11-12 sources in the sample may have redshift greater than 3.16 . 


\subsection{X-ray identifications}

The sample sources are identified with X-ray sources observed with the Imaging Proportional Counter (IPC) on the EINSTEIN observatory (Wilkes et al. 1994), and the Position Sensitive Proportional Counter (PSPC) on the ROSAT observatory. The latter data were taken from the "WGA" catalogue, and an updated version of the "First ROSAT source Catalogue of Pointed Observations". An $\mathrm{X}$-ray source is considered as a counterpart for a radio source if its normalized distance to the radio position is

$d=\left[\left(\Delta \alpha / \sigma_{\alpha}\right)^{2}+\left(\Delta \delta / \sigma_{\delta}\right)^{2}\right]^{1 / 2}<2.5$

where: $\Delta \alpha$ and $\Delta \delta$ are the differences between the $\mathrm{X}$ ray and radio position, and $\sigma_{\alpha}$ and $\sigma_{\delta}$ are the combined X-ray-radio position errors $\sigma_{i}=\left(\sigma_{\mathrm{X}-\text { ray }}^{2}+\sigma_{\text {rad }}^{2}\right)^{1 / 2}$ in right ascension and declination, respectively. Majority of the $\mathrm{X}$-ray sources are identified with compact radio sources (mostly quasars and BL Lac objects) for which accuracy of the VLA position is $\sigma_{\text {rad }} \approx 0.1-0.2$ arcsec.

Because the X-ray data available are not homogeneous, and the GB/GB2 sky region is only partly covered by the above X-ray observations, their details (epoch of observation, integration time, resultant counts, etc.) are not cited in this paper. Only a reference to the original data is given.

\section{Summary of the radio, optical, and X-ray data}

Radio, optical, and X-ray data for the sources in the revised GB/GB2 sample are summarized in Table 3, whose format is as follows:

Column 1: IAU-format source name.

Column 2: Sequence of 10 consecutive marks indicating whether the source appears in the following catalogues: (1) $151 \mathrm{MHz}$ : 6CII, III, VI (Hales et al. 1993; and references therein), (2) $178 \mathrm{MHz}$ : 4C (Pilkington \& Scott 1965; Gower et al. 1967), (3) $365 \mathrm{MHz}$ : Texas (Douglas et al. 1980), (4) 408 MHz: B2 (Colla et al. 1972; 1973), (5) B3 (Ficarra et al. 1985), (6) 750 and 1400 MHz: NRAO (Pauliny-Toth et al. 1966), (7) 966 MHz: Jodrell Bank (Cohen et al. 1977; Porcas et al. 1980), (8) $1400 \mathrm{MHz}$ : 83GB (Condon \& Broderick 1985; White \& Becker 1992), (9) $4830 \mathrm{MHz}$ : MGII (Langston et al. 1990); MGIV (Griffith et al. 1991), (10) $4850 \mathrm{MHz}$ GB6 (Gregory et al. 1996). "**-presence; "/"-absence; "c"-flux contaminated by confusing source or sources; "r"-source resolved, flux underestimated.

Column 3: "1", "2", and "3" denotes that the source belongs to Subsample 1, 2, and 3, respectively. "1, 2" denotes sources with $S \geq 0.25 \mathrm{Jy}$ in the area of $0.0906 \mathrm{sr}$, and " 2,3 " denotes sources with $0.20 \mathrm{Jy} \leq S<0.55 \mathrm{Jy}$ in the area of $0.0550 \mathrm{sr}$.

Column 4: Weighted mean 1.4-GHz flux density and its rms error (in mJy). " $v$ " denotes the standard deviation of variable flux (given in parentheses) instead of error of the mean.
Column 5: Slope of the fitted spectrum at $1.4 \mathrm{GHz}$ and its rms error. The slope is the derivative of a function $y=a+b x+c x^{2}$ or $y=a+b x+d \cdot \exp ( \pm x)($ cf. Sect. 3.1). If no function could be fitted to the spectral data (generally for variable sources), a crude slope at $1.4 \mathrm{GHz}$ is given in parentheses.

Column 6: Type of radio spectrum (cf. Sect. 3.1).

Column 7: Radio morphology (cf. Sect. 3.2). FRI-edge-darkened double, FRII-edge-brightened double (Fanaroff \& Riley 1974); C-compact, unresolved; C(1s), $\mathrm{C}(2 \mathrm{~s})$-core-dominated compact with one-sided or twosided extended emission, respectively. "cc" appended to the type denotes that a compact core has been detected, "CSS"-compact steep-spectrum, "Di"-diffuse emission.

Column 8: Largest angular size in arcsec.

Column 9: Code concerning the VLA maps available. "1.4" and sometimes "4.9 indicate observing frequency, and upper-case letters - VLA configuration used for observations ("P" means partially completed VLA in 1980). An asterisk $\left(^{*}\right)$ denotes that no map is available, and the source is represented by fitted Gaussian components only. If a VLBI-scale structure has been observed in the compact source, this is indicated.

Column 10: Source name repeated.

Column 11: Optical type. GAL — galaxy confirmed spectroscopically and/or with an extended image; (GAL) — possibly a galaxy; GAL? - identification ambiguous; QSO - quasar confirmed spectroscopically; BL - BL Lac object; RSO, NSO, BSO - red, neutral, and blue stellar object, respectively (most of NSOs and BSOs are quasar candidates); $\mathrm{RO}, \mathrm{BO}$ - red, and blue object too faint to be recognized as stellar or extended. EF — "empty field" source, an identification likely beyond the POSS limit. "X" preceding the type indicates that an X-ray source coincides with the radio position.

Column 12: Redshift. A value in parentheses has been estimated from the apparent magnitude and colour (cf. Sect. 4.2).

Columns 13 and 14: $R$ - and $B$-magnitudes. Two decimal digits precision indicate magnitudes measured with photoelectric or CCD photometry.

Columns 15 and 16: J(2000) sky coordinates. "o" preceding right ascension denotes the optical object's position. For double source without a radio core, the given position corresponds to a radio centroid.

Column 17: References to the large-scale radio structure and VLA maps, VLBI-scale structure, photometry, and redshift.

Column 18: "+" marks that a note to the source is appended.

Table 3. The revised GB/GB2 sample (The table is attached separately). 


\section{The statistics}

\subsection{Spectral content}

The spectral index at $1.4 \mathrm{GHz}, \alpha_{1.4}$, besides the total flux density at this frequency, is one of the best determined parameters of the GB/GB2 sources.

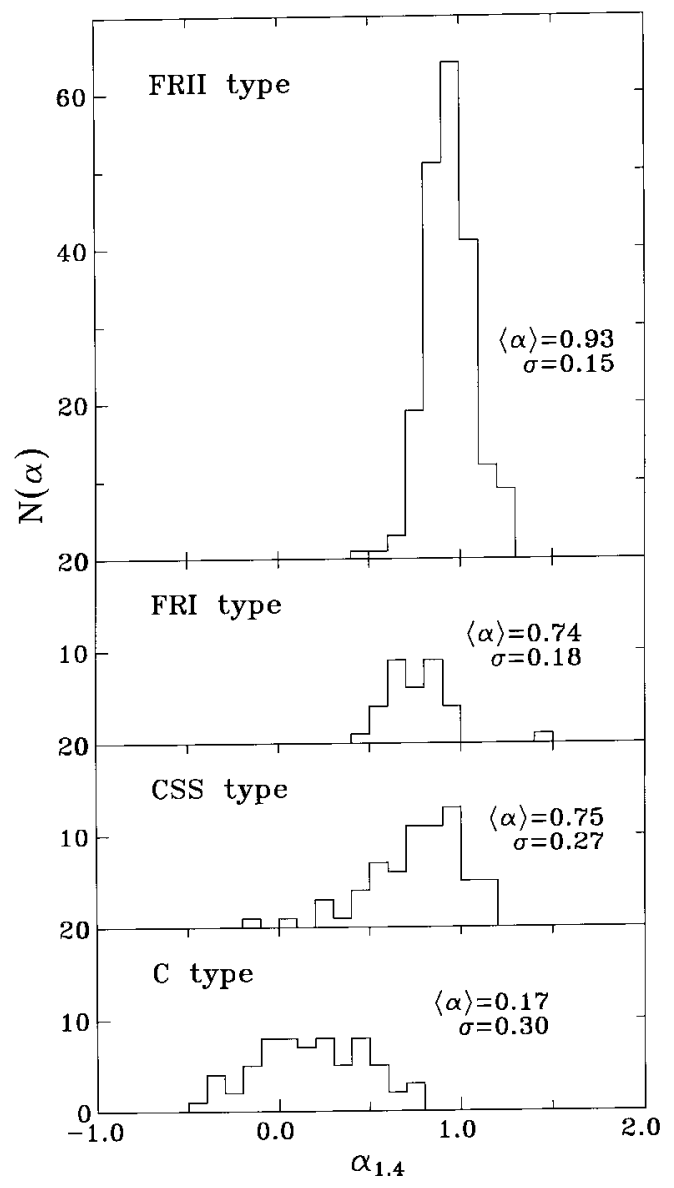

Fig. 1. Spectral-index $\alpha_{1.4}$ distributions of sources of different morphological type. $\alpha_{1.4}$ is a slope of the fitted spectrum at $1.4 \mathrm{GHz}$ (cf. Sect. 3.1). Their mean values and standard deviations are indicated

The distribution of $\alpha_{1.4}$, for four different morphological types is shown in Fig. 1. The distribution of $\alpha_{1.4}$ for different optical classes: galaxies, QSOs, and undetected (EF) sources, is shown in Fig. 2.

The mean values and standard deviations of these distributions are given on the relevant histograms. The standard deviations of $\alpha_{1.4}$ are always smaller than the corresponding deviation of any two-point spectral index.

The distributions of $\alpha_{1.4}$ obtained for the FRII and FRI as well as $\mathrm{C}$ sources are highly symmetrical. Momental skewness of the $\alpha_{1.4}$ distributions of FRII and FRI sources taken altogether is 0.036 ; the same of the

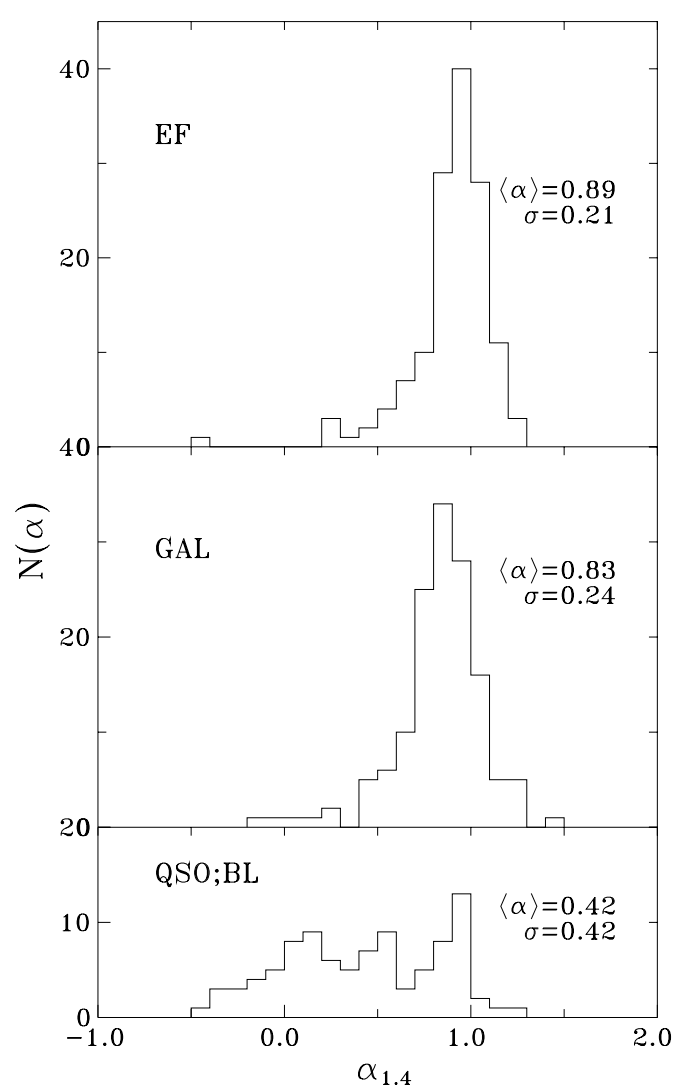

Fig. 2. The same as in Fig. 1 but for sources of different optical type

$\mathrm{C}$ sources is only 0.007. In turn, the large skewness of 1.089 for the CSS sources is evidently caused by different morphological types (small projected doubles, very compact self-absorbed sources, steep-spectrum core-jet structures, and complex sources which do not fit into any of the above categories) constituting the entire CSS class (e.g. Sanghera et al. 1995). The highly symmetrical spectral-index distributions of the lobe-dominated and core-dominated sources support the assumption about a Gaussian functional form which were frequently used to describe these distributions in cosmological evolutionary models (cf. Petrosian \& Dickey 1973; Kulkarni 1978; Condon 1984).

\subsection{Optical identification content}

For some GB/GB2 sources the optical type is uncertain. This is the case with the faintest objects barely visible on the POSS prints or detected by the CCD imaging beyond the POSS limit. Nevertheless for the statistical purpose, taking also into account their radio morphology and spectrum, one can include them into one of the two main categories: galaxy or quasar. Such a simplified optical identification content of the sample is given in Table 4. For each 
flux density range, the first row gives the fractions of all available identifications; the second - the corresponding fractions if optical identification is limited to $R<20$ mag (the POSS limit).

Table 4. The optical type vs. flux density range, cf. the text

$\begin{array}{llccc}\Delta S(\mathrm{Jy}) & \text { Galaxy } & \text { QSO } & \text { EF } & \text { sum } \\ & & & \\ >2.0 & 15(63 \% \pm 10 \%) & 7(29 \% \pm 9 \%) & 2(8 \% \pm 6 \%) & 24 \\ & 11(46 \% \pm 10 \%) & 6(25 \% \pm 9 \%) & 7(29 \% \pm 9 \%) \\ 0.55-2.0 & 84(40 \% \pm 4 \%) & 54(26 \% \pm 3 \%) & 71(34 \% \pm 4 \%) & 209 \\ & 62(30 \% \pm 4 \%) & 45(22 \% \pm 3 \%) & 102(49 \% \pm 7 \%) \\ 0.25-0.55 & 33(30 \% \pm 5 \%) & 27(25 \% \pm 4 \%) & 49(45 \% \pm 5 \%) & 109 \\ & 15(14 \% \pm 4 \%) & 21(19 \% \pm 4 \%) & 73(67 \% \pm 5 \%) \\ 0.20-0.25 & 8(26 \% \pm 8 \%) & 7(23 \% \pm 8 \%) & 16(52 \% \pm 9 \%) & 31 \\ & 5(16 \% \pm 7 \%) & 6(19 \% \pm 8 \%) & 20(65 \% \pm 10 \%)\end{array}$

The fraction of QSO identifications is similar in the different flux-limited subsamples; a decrease of the fraction with flux density is statistically unsignificant. Limiting the optical identification to $R<20 \mathrm{mag}$, this fraction decreases by about 4 per cent only in each flux range. The above is consistent with the independence of the radio properties of radio-loud quasars on their optical luminosities (e.g. Peacock et al. 1986). Some enlargement of the

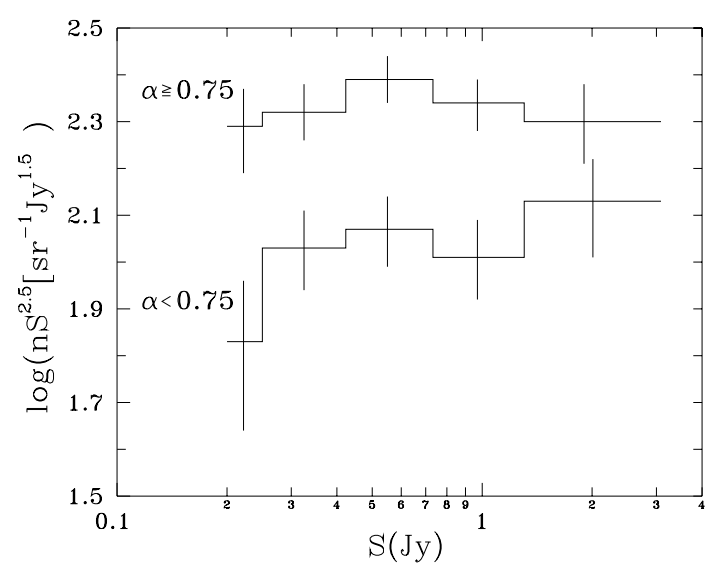

Fig. 3. Normalized differential counts of "steep-spectrum" and "flat-spectrum" GB/GB2 sources at $1.4 \mathrm{GHz}$

QSO fraction is expected after further identifications of the EF sources. Basing on the optical luminosity function of quasars and their radio-to-optical luminosity ratio function (cf. Machalski 1996), about $25 \pm 5$ more QSOs with $R \geq 20 \mathrm{mag}$ and $S_{1.4}>200 \mathrm{mJy}$ can be expected. Furthermore, a decrease of the galaxy identifications with decreasing flux density limit is evident. Therefore, most of the EF sources should be distant powerful galaxies. A distribution of the optical type for different radio morphologies is shown in Table 5.

Table 5. The optical type vs. radio morphology

$\begin{array}{lrrrr}\text { Morph. } & \text { Galaxy } & \text { QSO } & \text { EF } & \text { sum } \\ & & & & \\ \text { FRII } & 85(42 \% \pm 5 \%) & 26(13 \% \pm 2 \%) & 92(45 \% \pm 4 \%) & 203 \\ \text { FRI } & 29(85 \% \pm 6 \%) & 2(6 \% \pm 4 \%) & 3(9 \% \pm 5 \%) & 34 \\ \text { CSS } & 19(28 \% \pm 6 \%) & 11(16 \% \pm 5 \%) & 38(56 \% \pm 6 \%) & 68 \\ \text { C } & 5(8 \% \pm 3 \%) & 56(84 \% \pm 5 \%) & 5(8 \% \pm 3 \%) & 66 \\ \text { Disk } & 2 & & & 2\end{array}$

A majority of FRI type emission is connected to radio galaxies, while most of $\mathrm{C}$ types, frequently with onesided (1s) or two-sided (2s) emission detected besides the bright core, are related to QSOs. However, there is no clear distinction between FRI and large $\mathrm{C}+2 \mathrm{~s}$ radio sources. Usually a linear extent of $\mathrm{C}+1 \mathrm{~s}$ or $\mathrm{C}+2 \mathrm{~s}$ structure does not exceed the size of a parent optical object, i.e. about $10-15 \mathrm{kpc}$. However, in some quasars twosided emission extends over much larger distances from the core; these are classified here as FRI (e.g. 0827+378, $1148+387)$. Concerning the FRII sources, we estimate that no more than 10 per cent of EF sources in the sample can be quasars; the remaining ones should be distant galaxies. The compact steep-spectrum (CSS) sources are found both in galaxies and QSOs. The relatively large fraction of CSS EF sources suggests that they are very distant.

\subsection{Source counts}

\subsubsection{The spectrum-dependent counts}

The counts of all radio sources at $1.4 \mathrm{GHz}$ are very well established down to a sub-mJy level (e.g. Windhorst et al. 1985). The first limited spectral counts at this frequency were published by Machalski (1978b), but over almost two decades had remained unimproved, and were not used, for example, to constrain the cosmological models. However, such constraints at 2.7 and $5 \mathrm{GHz}$ were successfully applied to the evolutionary models of Condon (1984) and Dunlop \& Peacock (1990).

Although dividing of sources into "flat-spectrum" and "steep-spectrum" populations, in the face of "unified models", is now by large unjustified; spectrum-dependent counts can be still useful for cosmological purposes. For these purposes, the source population can be separated into two subpopulations with an arbitrarily chosen spectral index. Such differential counts of the sample sources with $\alpha_{1.4}<0.75$ and $\alpha_{1.4} \geq 0.75$, normalized to the Euclidean ones, are shown in Fig. 3. Numerical data of these counts, extended to a lower flux density limit on the basis of other $1.4-\mathrm{GHz}$ samples, will be published in 
a forthcoming paper (Machalski \& Jamrozy, in preparation).

\subsubsection{The radio type-dependent counts}

There are already indications that the spatial distributions of sources of different morphological type are not identical, i.e. these sources could evolve differently in cosmic time. While there is no doubt that the powerful radio galaxies and quasars show a strong evolution; the amount of evolution of low-power sources (mJy-level radio galaxies, Seyferts, etc.) is still controversial. Even among powerful

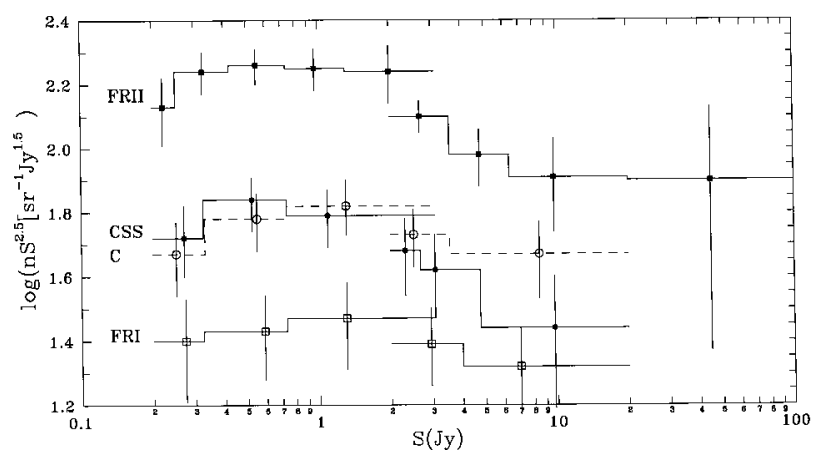

Fig. 4. Normalised differential counts of sources of different morphological type at $1.4 \mathrm{GHz}$

sources the amount of cosmological evolution may not be the same for separate types (e.g. FRI and FRII).

In order to enable the use of the GB/GB2 data for exploring the implications of unified-model schemes, the normalised differential counts of the FRII, FRI, CSS, and C sources are provided in Fig. 4.

After extending these counts to the flux density of $100 \mathrm{Jy}$ (taking into account the sources with $2 \mathrm{Jy}<S_{1.4}<100 \mathrm{Jy}$ in the sky area of $4.22 \mathrm{sr} ; \delta>10^{\circ},\left|b_{\text {II }}\right| \geq 10^{\circ}$ ) it can be seen that the counts of FRI sources to the flux limit of $0.2 \mathrm{Jy}$ are much flatter than the corresponding counts of FRII sources. Also the counts of the CSS and C sources probably differ between themselves, although the statistics available here is not sufficient to prove this. A further study of the above counts over larger sky areas is in progress (Machalski \& Jamrozy, in preparation).

\subsection{Redshift distributions}

Complete redshift data of a given sample of radio sources are highly required for calculations of their intrinsic power, linear size, etc. Such data are also crucial for the observational verification of cosmological evolutionary models. Unfortunately, completing spectroscopic redshift content of most of the flux-limited samples is very difficult for the well known reasons. However, for some objects, e.g. elliptical radio galaxies, redshift can be reliably estimated from their apparent magnitude (especially $\mathrm{K}$ mag) and/or angular size. The latter method was used by Vigotti et al. (1989) to estimate galaxy redshifts in the B3-VLA sample.

In this paper, another possibility of estimating a redshift distribution of the subsamples of FRII sources is employed. The estimation is based on the empirical correla-

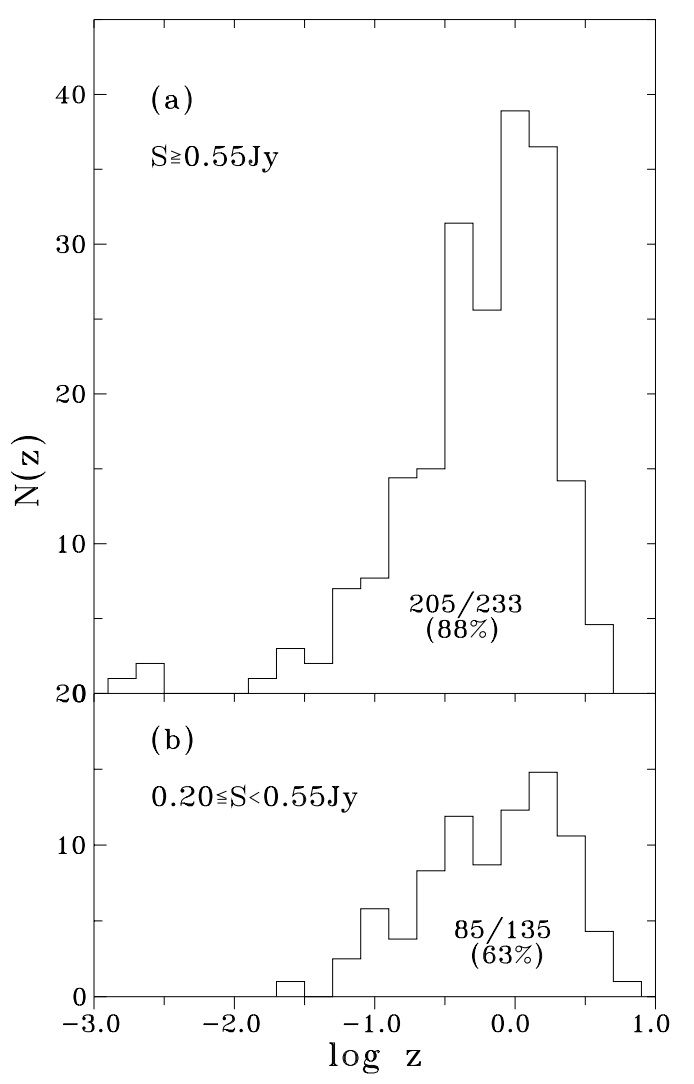

Fig. 5. Estimated redshift distributions of sources: a) in Subsample 1; b) in Subsample $2+3$. The fraction of redshifts measured and/or estimated in each Subsample is given

tion between the power and surface brightness $(\log P$ vs. $\log B)$ of FRII-type sources; the latter parameter being dependent on the apparent flux density and angular separation between hot spots in their lobes. It can be shown (Machalski, in preparation) that for a given $\log B$ (a range of $\log B$, in practice), the distribution of $\log P$ has a definite functional form. Therefore, for each FRII source in a sample one can calculate the probability of having $\log P$ in a certain power range (bin), and hence a redshift range. A sum of these probabilities in each redshift range, divided by the number of sources involved, gives the normalised distribution of redshift. If the sample is unbiased (the implicit assumption about random sampling of the redshift is present), the estimated $z$-distribution is statistically 
Table A1. Gaussian models for the added sources. The components are fitted to our own, or FIRST [ddddd+dd], or NVSS [CddddPdd] VLA maps

\begin{tabular}{|c|c|c|c|c|c|c|c|c|c|c|}
\hline $\begin{array}{l}\text { GB/GB2 } \\
\text { name }\end{array}$ & & $\begin{array}{r}\text { R.A. } \\
\text { (J2000) }\end{array}$ & & $\begin{array}{r}\text { Decl. } \\
(\mathrm{J} 2000)\end{array}$ & $\begin{array}{r}S_{1.4} \\
{[\mathrm{~mJ} \mathrm{y}]}\end{array}$ & $\begin{array}{r}\text { Comp. size } \\
{\left[{ }^{\prime \prime}\right] \times\left[^{\prime \prime}\right]}\end{array}$ & $\begin{array}{l}\text { PA } \\
{[0]}\end{array}$ & $\begin{array}{r}\mathrm{LAS} \\
{\left[{ }^{\prime \prime}\right]}\end{array}$ & $\begin{array}{l}\text { OPA } \\
{[0]}\end{array}$ & $\begin{array}{l}\text { VLA } \\
\text { map }\end{array}$ \\
\hline $0753+360$ & & $\begin{array}{r}075621.56 \\
21.98\end{array}$ & +35 & $\begin{array}{r}5443.0 \\
41.0\end{array}$ & $\begin{array}{l}204 \\
208\end{array}$ & $\begin{array}{l}2.8 \times 1.9 \\
3.0 \times 1.9\end{array}$ & $\begin{array}{ll}13 & 8 \\
113 & 3\end{array}$ & 8.3 & 111 & $07570+36$ \\
\hline $0753+373$ & opt. & $\begin{array}{r}075628.26 \\
28.26\end{array}$ & +37 & $\begin{array}{r}1455.8 \\
53.0\end{array}$ & 236 & $1.5 \times 0.8$ & 35 & - & - & $07570+37$ \\
\hline $0754+368$ & opt. & $\begin{array}{r}075751.29 \\
52.63 \\
53.82 \\
53.34\end{array}$ & $\begin{array}{r}+36 \\
4\end{array}$ & $\begin{array}{cc}39 & 33.6 \\
40 & 12.5 \\
40 & 42.2 \\
40 & 19.6\end{array}$ & $\begin{array}{r}130 \\
5 \\
162\end{array}$ & $\begin{array}{l}43 \times 19 \\
45 \times 11\end{array}$ & $\begin{array}{r}16 \\
- \\
7\end{array}$ & $>76$ & 24 & C0806P36 \\
\hline $0809+503$ & opt. & $\begin{array}{rr}081317.11 \\
& 20.55 \\
& 18.90\end{array}$ & +50 & $\begin{array}{r}1229.1 \\
47.1 \\
41.9\end{array}$ & $\begin{array}{l}225 \\
343\end{array}$ & $\begin{array}{r}19 \times 14 \\
19 \times<13\end{array}$ & $\begin{array}{ll}6 & 2 \\
5 & 3\end{array}$ & 47 & 61 & C0824P52 \\
\hline $0813+379$ & & $\begin{array}{r}081644.52 \\
45.01\end{array}$ & +37 & $\begin{array}{r}4912.1 \\
20.1\end{array}$ & $\begin{array}{ll}13 & 1 \\
19 & 0\end{array}$ & $\begin{array}{l}3.3 \times 1.3 \\
4.1 \times 1.2\end{array}$ & $\begin{array}{l}25 \\
42\end{array}$ & 12 & 126 & $08150+37$ \\
\hline $0820+367$ & & $\begin{array}{r}082347.83 \\
48.62\end{array}$ & +36 & $\begin{array}{r}3251.6 \\
31.1\end{array}$ & $\begin{array}{r}180 \\
74\end{array}$ & $\begin{array}{l}1.6 \times 0.7 \\
3.0 \times 1.5\end{array}$ & $\begin{array}{r}139 \\
0\end{array}$ & 23 & 156 & $08240+36$ \\
\hline $0922+322$ & & 092532.73 & +31 & 5952.9 & 531 & $3.6 \times 1.4$ & 166 & - & - & $09240+32$ \\
\hline $0958+346$ & opt. & $\begin{array}{rr}10 & 01 \\
& 11.92 \\
& 11.95\end{array}$ & +34 & $\begin{array}{r}2450.4 \\
49.5\end{array}$ & 203 & $0.6 \times 0.3$ & 29 & $<1.2$ & - & own \\
\hline $1042+392$ & & $\begin{array}{r}104514.67 \\
\\
15.40\end{array}$ & +38 & $\begin{array}{r}5639.8 \\
36.7\end{array}$ & $\begin{array}{ll}31 & 6 \\
35 & 0\end{array}$ & $\begin{array}{l}2.3 \times 0.8 \\
1.9 \times 1.0\end{array}$ & $\begin{array}{l}65 \\
85\end{array}$ & 11 & 96 & own \\
\hline $1127+348$ & & $\begin{array}{r}113004.95 \\
06.57\end{array}$ & +34 & $\begin{array}{r}3440.6 \\
24.3\end{array}$ & $\begin{array}{r}95 \\
102\end{array}$ & $\begin{array}{l}24 \times 15 \\
19 \times 13\end{array}$ & $\begin{array}{l}130 \\
177\end{array}$ & 35 & 127 & $11300+34$ \\
\hline $1420+326$ & opt. & $\begin{array}{r}142230.38 \\
30.35\end{array}$ & +32 & $23 \begin{array}{r}10.4 \\
10.0\end{array}$ & 408 & $1.3 \times 1.0$ & 18 & - & - & $14210+32$ \\
\hline $1550+346$ & & 155250.59 & +34 & 3016.3 & 195 & $1.2 \times 1.1$ & 90 & - & - & $15510+34$ \\
\hline $1619+378$ & opt. & $\begin{array}{rr}1621 & 11.29 \\
& 11.43\end{array}$ & +37 & $\begin{array}{r}4604.9 \\
04.7\end{array}$ & 627 & $1.0 \times 0.8$ & 55 & - & - & $16210+37$ \\
\hline
\end{tabular}

consistent with the distribution of true, spectroscopic redshift.

Using this method, the redshift distributions obtained for 88 per cent of the sources in Subsample 1 is shown in Fig. 5a. The sources for which the redshift cannot be estimated are exclusively compact ones and mostly optically unidentified. Similarly, the estimated redshift distribution for 63 per cent of the sources in Subsamples 2 and 3 is shown in Fig. 5b. These distributions suggest that about 8-10 FRII-type sources in our sample can be expected at redshift $z>3$. The number of objects above this redshift may be even greater if the unidentified compact sources (especially CSS ones) are taken into account.

\section{Appendix}

This Appendix contains additional $1400-\mathrm{MHz}$ and $4885-\mathrm{MHz}$ VLA maps of some sources from the original sample, and numerical data of those sources included in the revised sample which are adequately described by Gaussian component fits to the VLA images.

Figure A1 shows the $4885-\mathrm{MHz}$ maps of central regions of six but one radio galaxies from Table 4 . The maps resolution (FWHB of the restoring beam) is about 0.5 arcsec. Two of these galaxies $(0912+489$ and $1104+365)$ have FRII morphology with unresolved radio cores. The remaining $(0838+325,1059+351,1141+466$, and $1325+321)$ are classified as FRI type. In these latter sources, a radio core was detected in the $0838+325$ galaxy only. The inner structure of this galaxy, of size of about $17 \mathrm{kpc}$, consists of two bright emitting regions extended symmetrically off the core. They resemble usual "hot spots" at the edge of classical double edge-brigthtened (FRII) structure, however the low-resolution 1.4-GHz VLA images of this source (Fanti et al. 1986; 1987) show very extended diffuse emission stretched out far away from these bright spots, and characteristic for edge-darkened sources. No trace of jets were detected. A similar inner 
structure but without a pronounced core characterizes the galaxy $1141+466$ as well. In turn, the brightest spots in an inner "S-shaped" structure of the galaxies 1059+351 and $1325+321$ are evidently related to a base of twisted jets.

The inner part of the optically unidentified source $1348+352$ is very enigmatic. The VLA $1465-\mathrm{MHz}$ map (Machalski \& Condon 1983b) showed the very bright central region suggesting a core-jet structure and a lowbrightness extended emission. A twisted ridge could be seen in it. The reality of that emission is now confirmed due to the FIRST survey. A relevant image of the source (on the FIRST map 13510+35071) indicates that the diffused emission includes about 45 per cent of the total flux at $1400 \mathrm{MHz}$. Surprisingly, our VLA 4885-MHz map (Fig. A1) of the source's central region with the angular resolution of about 0.5 arcsec does not show a typical core structure. The $3 \times 2.5$ arcsec region reveals a "hot spot" at its westernmost side and a ring-like emission towards the east. This "hot spot" with a deconvolved size of $0.7 \times 0.6$ arcsec has a spectral index between 1.4 and $5 \mathrm{GHz}, \alpha_{1.4}^{5} \approx 0.67 \pm 0.06$. It is flatter than the corresponding index of the central region, which has $\alpha_{1.4}^{5}=0.84 \pm 0.04$. The extended diffuse emission has exactly the same index of 0.84 . Consequently, the source $1348+352$ is probably of FRI type, with twisted structure characteristic for precessing jets but strongly projected onto the sky.

The correct $1400-\mathrm{MHz}$ map of the galaxy $0910+353$, reproduced from the FIRST $09120+35$ map is shown in Fig. A2. Parameters of the Gaussian components fitted by the least-square method to VLA images of the sources added to the revised sample, and the sky coordinates of the identified optical objects (cf. Table 3), are given in Table A1. If these fits were taken from the FIRST or NVSS maps, this is noted in Table A1 by the number of original VLA map.

\section{References}

Allington-Smith J.R., Perryman M.A.C., Longair M.S., Gunn J.E., 1982, MNRAS 201, 331

Allington-Smith J.R., Spinrad H., Djorgovski S., Liebert J., 1988, MNRAS 234, 1091

Andernach H., Waldthausen H., Wielebinski R., 1980, A\&AS 41, 339

Antonucci R.R.J., 1985, ApJS 59, 499

Baars J.W.M., Genzel R., Pauliny-Toth I.I.K., Witzel A., 1977, A\&A 61,99

Becker R.H., White R.L., Helfand D.J., 1995, ApJ 450, 559

Benn C.R., Grueff G., Vigotti M., Wall J.V., 1982, MNRAS 200, 747

Burbidge E.M., 1970, ApJ 160, L33

Burbidge E.M., Strittmatter P.A., 1972, ApJ 172, L37

Burns J.O, Schwendeman E., White R.A., 1983, ApJ 271, 575

Capetti A., Morganti R., Parma P., Fanti R., 1993, A\&AS 99, 407

Colla G., Fanti C., Fanti R., et al., 1972, A\&AS 7, 1
Colla G., Fanti C., Fanti R., et al., 1973, A\&AS 11, 291

Cohen A.M., Porcas R.W., Browne I.W.A., Daintree E.J., Walsh D., 1977, MmRAS 84, 1

Condon J.J., 1983, ApJS 53, 459

Condon J.J., 1984, ApJ 287, 461

Condon J.J., Broderick J.J., 1985, AJ 90, 2540

Condon J.J., Broderick J.J., 1988, AJ 96, 30

Condon J.J., Broderick J.J., Seielstad,G.A., 1989, AJ 87, 1064

Condon J.J., Cotton W.D., Greisen E.W., et al., 1996, NCSA Astr. Dig. Image Lib.

Cousins A.W.J., 1976, MmRAS 81, 25

Crawford C.S., Fabian A.C., 1996, MNRAS 282, 1483

deRuiter H.R., Parma P., Fanti C., Fanti R., 1986, A\&AS 65, 111

Dixon R.S., Kraus J.D., 1968, AJ 73, 381

Djorgovski S.G., Thompson D.J., Vigotti M., Grueff G., 1990, PASP 102, 113

Douglas J.N., Bash F.N., Torrence G.W., Wolfe C., 1980, Univ. of Texas, Prep. No. 17

Dunlop J.S., Peacock J.A., 1990, MNRAS 247, 19

Fanaroff B.J., Riley J.M., 1974, MNRAS 167, 31P

Fanti C., Fanti R., Gioia I.M., et al., 1977, A\&AS 29, 279

Fanti C., Fanti R., deRuiter H.R., Parma P., 1986, A\&AS 65, 145

Fanti C., Fanti R., deRuiter H.R., Parma P., 1987, A\&AS 69, 57

Fey AL., Clegg A.W., Fomalont E.B., 1996, ApJS 105, 299

Ficarra A., Grueff G., Tomassetti G., 1985, A\&AS 59, 255

Ford H., Crane P., Jacoby G.H., Lawrie D.G., van der Hulst J.M., 1985, ApJ 293, 132

Gioia I.M., Fabbiano G., 1987, ApJS 63, 771

Gower J.F.R., Scott P.F., Wills D., 1967, MmRAS 71, 49

Gregory P.C., Scott W.K., Douglas K., Condon J.J., 1996, ApJS 103, 427

Griffith M., Langston G., Heflin M., Conner S., Burke B., 1991, ApJS 75, 801

Grueff G., Vigotti M, 1979, A\&AS 35, 371

Grueff G., Vigotti M., Spinrad H., 1980, A\&A 86, 50

Hales S.E.G., Baldwin J.E., Warner P.J., 1993, MNRAS 263, 25

Haslam C.G.T., Kronberg P.P., Waldthausen H., Wielebinski R., 1978, A\&AS 31, 99

Henstock D.R., Browne I.W.A., Wilkinson P.N., et al., 1995, ApJS 100, 1

Henstock D.R., 1995 (priv. info.)

Hewitt A., Burbidge G., 1993, ApJS 87, 451 (QSO cat.)

Hook I.M., MaMahon R.G., Irwin M.J., Hazard C., 1996, MNRAS 282, 1274

Huchra J., Davis M. Latham D., Tonry J., 1983, ApJS 52, 89

Hughes P.A., Aller H.D., Aller M.F., 1992, ApJ 396, 469

Hummel E., Pedlar A., van der Hulst J.M., Davies R.D., 1985, A\&AS 60, 293

Jaffe W., Perola G.C., 1974, A\&A 31, 223

Johnson H.L., 1966, ARA\&A 4, 193

Katgert J.K., 1978, A\&AS 31, 409

Katgert P., Katgert-Merkelijn J.K., LePoole R.S., van der Laan H., 1973, A\&A 23, 171

Katgert-Merkelijn J., Lari C., Padrielli L., 1980, A\&AS 40, 91 Kulkarni V.K., 1978, MNRAS 185, 123

Kühr H., Nauber U., Pauliny-Toth I.I.K., Witzel A., 1981, A\&AS 45, 367 
LaHulla J.F., Merighi R., Vettolani G., Vigotti M., 1991, A\&AS 88, 525

Laing R.A., Riley J.M., Longair M.S., 1983, MNRAS 204, 151

Langston G.I., Heflin M.B., Conner S.R., et al., 1990, ApJS 72,621

Leahy J.P., Perley R.A., 1991, AJ 102, 537

Lilly S.J., Longair M.S., Allington-Smith J.R., 1985, MNRAS 215,37

Lousdale C.J., Barthel P.D., Miley G.K., 1993, ApJS 87, 63

Machalski J., 1978a, Acta Astr. 28, 367

Machalski J., 1978b, A\&A 65, 157

Machalski J., 1988, Acta Astr. 38, 163

Machalski J., 1991, Acta Astr. 41, 39

Machalski J., 1992, Acta Astr. 42, 335

Machalski J., 1996, in "Extragalactic Radio Sources" (IAU Symp. No. 175), Ekers R. et al. (ed.). Kluwer Acad. Pub., p. 545

Machalski J., Brandt W.N., 1996, MNRAS 282, 1305

Machalski J., Condon J.J., 1983a, AJ 88, 143 (Paper III)

Machalski J., Condon J.J., 1983b, AJ 88, 1591 (Paper IV)

Machalski J., Condon J.J., 1985a, AJ 90, 5 (Paper V)

Machalski J., Condon J.J., 1985b, AJ 90, 973

Machalski J., Condon J.J., 1986, AJ 91, 998

Machalski J., Condon J.J., 1990, PASP 102, 53

Machalski J., Engels D., 1994, MNRAS 266, L69

Machalski J., Inoue M., 1990, MNRAS 243, 209

Machalski J., LaFranca F., 1988, IAU Circ. No. 4573

Machalski J., Magdziarz P., 1993a, A\&A 267, 363

Machalski J., Magdziarz P., 1993b, A\&AS 102, 315

Machalski J., Maslowski J., 1982, AJ 87, 1132 (Paper I)

Machalski J., Maslowski J., Condon J.J., Condon M.A., 1982, AJ 87, 1150 (Paper II)

Machalski J., Wiśniewski W.Z., 1988, MNRAS 231, 1065

Maslowski J., 1972, Acta Astr, 22, 227

Maslowski J., Kellermann K.I., 1988, AJ 95, 1659

Maxfield L., Thompson D., Djorgovski S., et al., 1995, PASP 107,369

Moles M., Garcia-Pelayo J.M., Masegosa J., Aparicio A., Quintana J.M., 1985, A\&A 152, 271

Morabito D.D., Niell A.E., Preston R.A., et al., 1986, AJ 91, 1038

Morganti R., Ulrich M.H., Tadhunter C.N., 1992, MNRAS 254, 546

Morganti R., Killeen N.E.B., Tadhunter C.N., 1993, MNRAS 263, 1023

Netzer H., Heller A., Loinger F., et al., 1996, MNRAS 279, 429

O'Donoghue A.A., Eilek J.A., Owen F.N., 1993, ApJ 408,428

Owen F.N., Condon J.J., Ledden J.E., 1983, AJ 88, 1

Owen F.N., Puschell J.J., 1984, AJ 89, 932

Owen F.N., White R.A., Thronson H.A., 1988, AJ 95, 1

Parma P., deRuiter H.R., Fanti C., Fanti R., 1986, A\&AS 64, 135

Pauliny-Toth I.I.K., Wade C.M., Heeschen D.S., 1966, ApJS 13,65

Peacock J.A., Miller L., Longair M.S., 1986, MNRAS 218, 265

Pearson T.J., Readhead A.C.S., 1988, ApJ 328, 114

Perley R.A., 1982, AJ 87, 859

Perley R.A., Fomalont E.B., Johnston K.J., 1980, AJ 85, 649

Perley R.A., Fomalont E.B., Johnston K.J., 1982, ApJ 255, L93

Petrosian V., Dickey J., 1973, ApJ 186, 403
Pilkington J.D.H., Scott P.F., 1965, MmRAS 69, 183

Polatidis A.G., Wilkinson P.N., Xu W., et al., 1995, ApJS 98, 1

Porcas R.W., Urry C.M., Browne I.W.A., et al., 1980, MNRAS 191, 607

Reid A., Shone D.L., Akujor C.E., et al., 1995, A\&AS 110, 213

Rioja M.J., Porcas R.W., Machalski J., 1996, Proc. IAU Symp. No. 175 "Extragalactic Radio Sources", Ekers R. et al. (ed.). Kluwer Acad. Pub., p. 122

Rudnick L., Owen F.N., 1977, AJ 82, 1

Ryś S., Machalski J., 1990, A\&A 236, 15

Sandage A., 1972, ApJ 178, 25

Sandage A., Kristian,J., Westphal,J.A., 1976, ApJ 205, 688

Sanghera H.S., Saikia,D.J., Lüdke,E., et al., 1995, A\&A 295, 629

Sargent W.L.W., 1973, ApJ 182, L13

Seielstad G.A., Pearson T.J., Readhead A.C.S., 1983, PASP 95,842

Smith H.E., Spinrad H., 1980, PASP 92, 553

Spinrad H., 1982, PASP 94, 397

Spinrad H., Djorgovski S.G., 1984, ApJ 285, L49

Spinrad H., Filippenko A.V., Wyckoff S., et al., 1985, ApJ 299, L7

Stickel M., Kühr H., 1994, A\&AS 105, 67

Strittmatter P.A., Carswell R.F., Gilbert G., Burbidge E.M., 1974, ApJ 190, 509

Strom S.E., Riley J.M., Spinrad H., et al., 1990, A\&A 227, 19

Tadhunter C.N., Morganti R., di Serego Alighieri S., Fosbury R.A.E., Danziger I.J., 1993, MNRAS 263, 999

Thakkar D.D., Xu W., Readhead A.C.S., et al., 1995, ApJS 98, 33

Thompson D., Djorgovski S., Vigotti M., Grueff G., 1992, ApJS 81,1

Vallée J.P., Wilson A.S., 1976, Nat 259, 451

Vallée J.P., Bridle A.H., Wilson A.S., 1981, ApJ 250, 66

Valtaoja E., Teräsranta H., Urpo S., et al., 1992, A\&A 254, 80

van Albada G.D., van der Hulst J.M., 1982, A\&A 115, 263

Véron-Cetty M.P., Véron P., 1993, ESO Sci. Report No. 13

Vermeulen R.C., Taylor G.B., Readhead A.C.S., Browne I.W.A., 1996, AJ 111, 1013

Vigotti M., Grueff G., Perley R., Clark B.G., Bridle A.H., 1989, AJ 98, 419

Waldthausen H., Haslam C.G.T., Wielebinski R., Kronberg P.P., 1979, A\&AS 36, 237

Wall J.V., Peacock J.A., 1985, MNRAS 216, 173

Walsh D., Wills B.J., Wills D., 1979, MNRAS 189, 667

Warner P.J., Riley J.M., Eales S.A., Downes A.J.B., Baldwin J.E., 1983, MNRAS 204, 1279

White R.L., Becker R.H., 1992, ApJS 79, 331

Wilkes B.J., Tananbaum H., Worrall D.M., et al., 1994, ApJS 92,53

Windhorst R.A., Miley G.K., Owen F.N., Kron R.G., Koo D.C., 1985, ApJ 289, 494

Wilkinson A., Hine R.G., Sargent W.L.W., 1981, MNRAS 196, 669

Worrall D.M., Wilkes B.J., 1990, ApJ 360, 396

$\mathrm{Xu}$ W., Reahead A.C.S., Pearson T.J., Polatidis A.G., Wilkinson P.N., 1995, ApJS 99, 297

Zwicky F., Herzog E., 1963, "Catalogue of Galaxies and Clusters of Galaxies", Caltech (ed.), Pasadena 

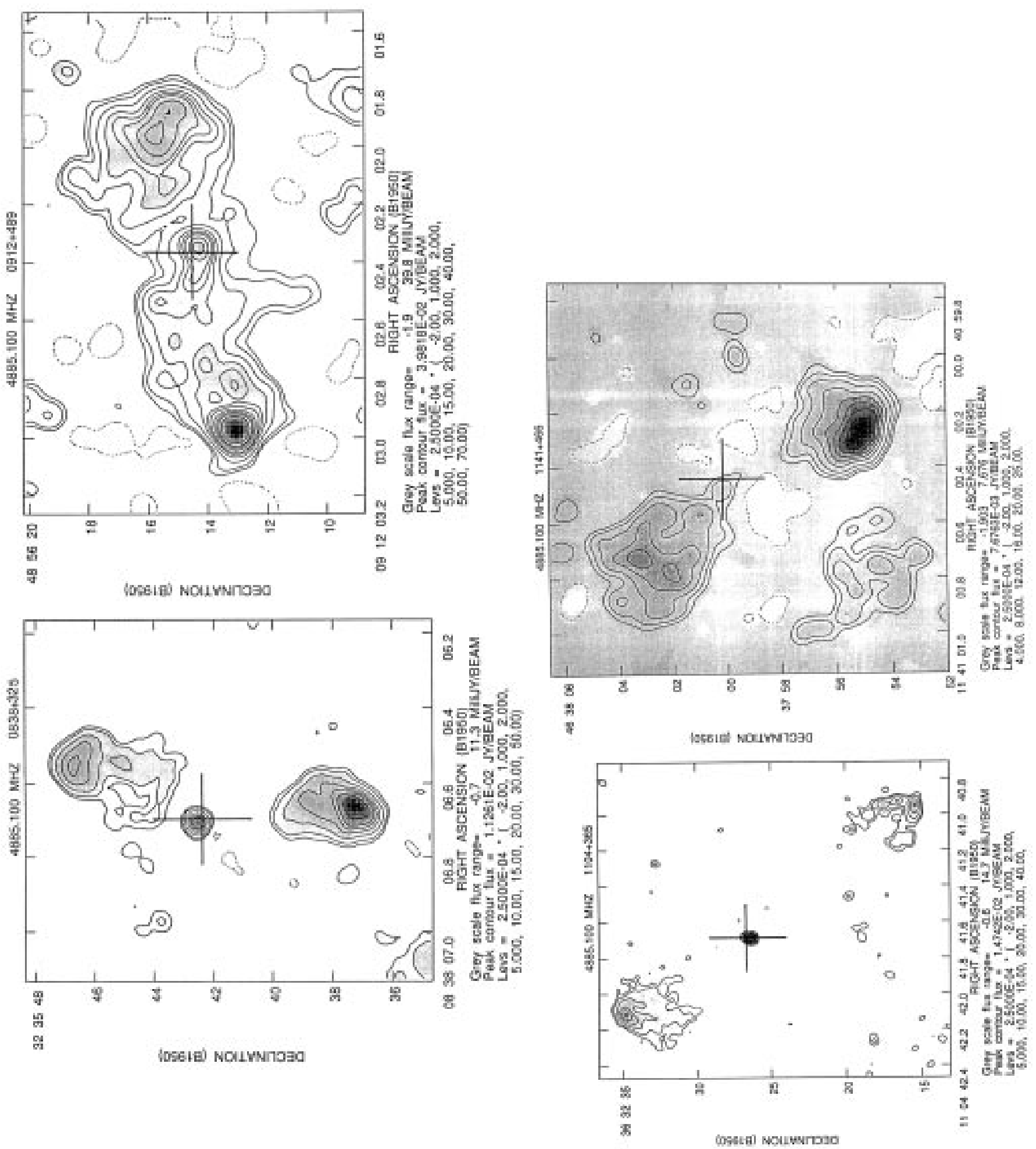

Fig. A1. VLA 4885-MHz contour maps of the sources selected from the revised GB/GB2 sample. FWHB of the restoring beam is $0.55 \times 0.50$ arcsec. The contour levels are shown under each map. The best optical positions of the center of identified galaxies are marked by the large crosses 

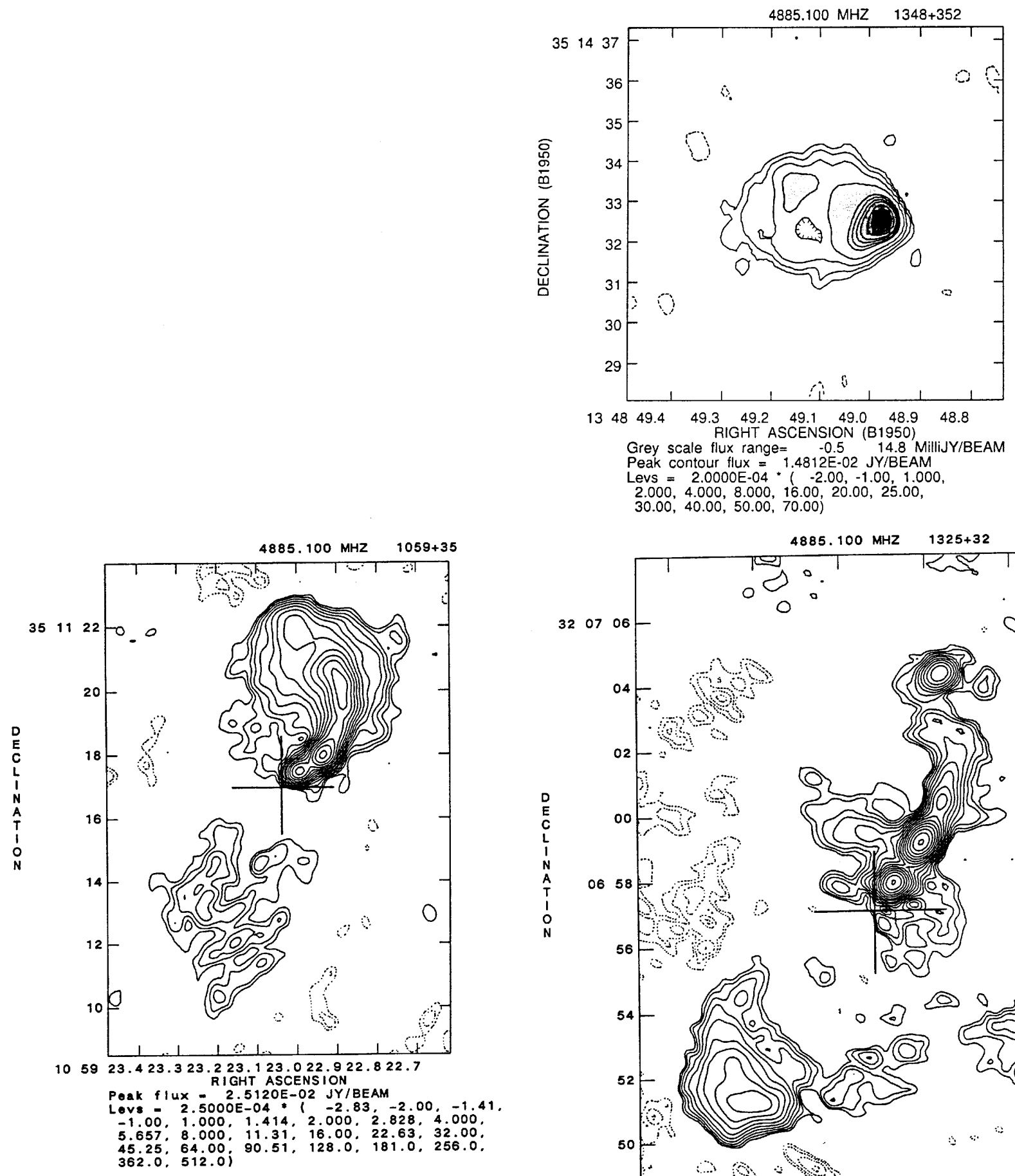

Grey scale flux range $=-0.5 \quad 14.8$ MilliJY/BEAM

Grey scale flux range $=140.5 \quad 14.8$ Mill

Leak $=2.0000 \mathrm{E}-04 *(-2.00,-1.00,1.000$

$2.000,4.000,8.000,16.00,20.00,25.00$

$30.00,40.00,50.00,70.00$ )

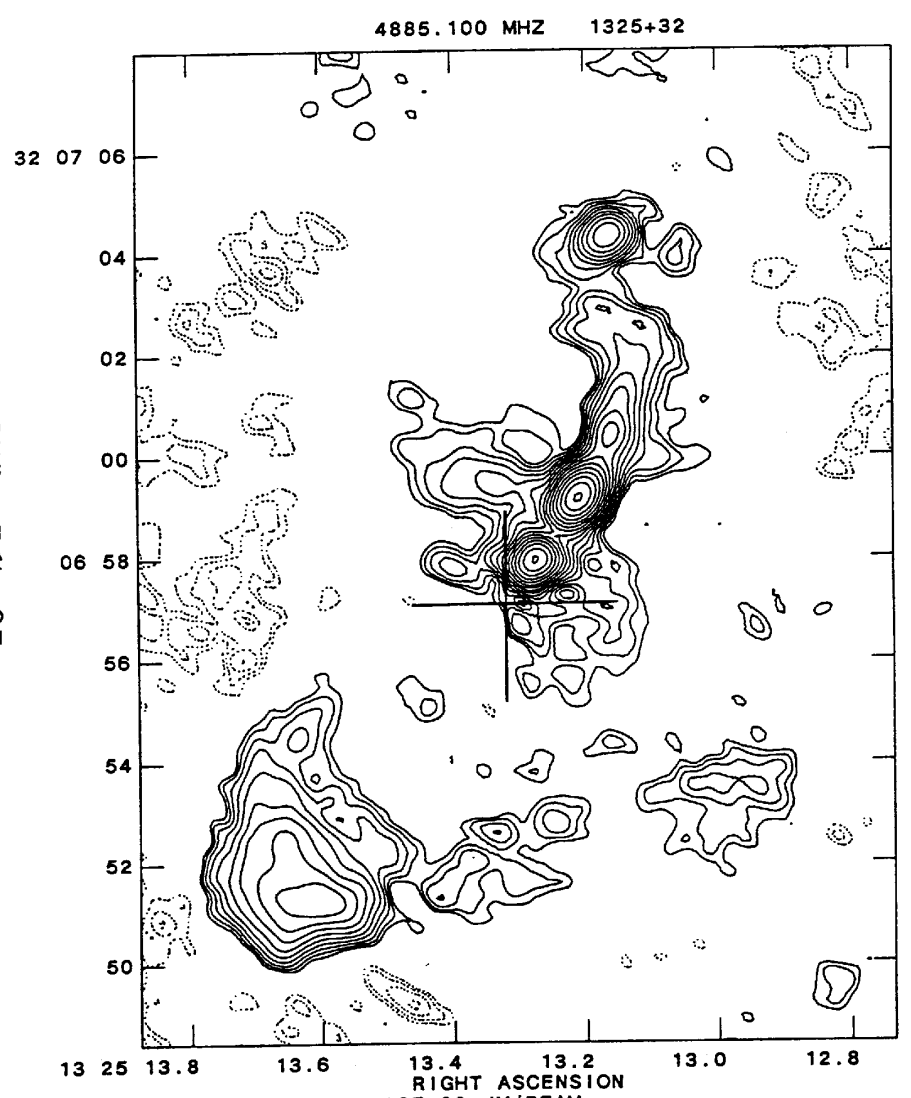

Peak fluX - 5.3129E-02 JY/BEAM

Lovs - 4.0000E-04:1 $-2.83,-2.00,-1.41$

$-1.00,1.000,1.414,2.000,2.828,4.000$.

$5.657,8.000,11.31,16.00,22.63,32.00$.

$45.25,64.00$
$362.0,512.0$

Fig. A1. continued 


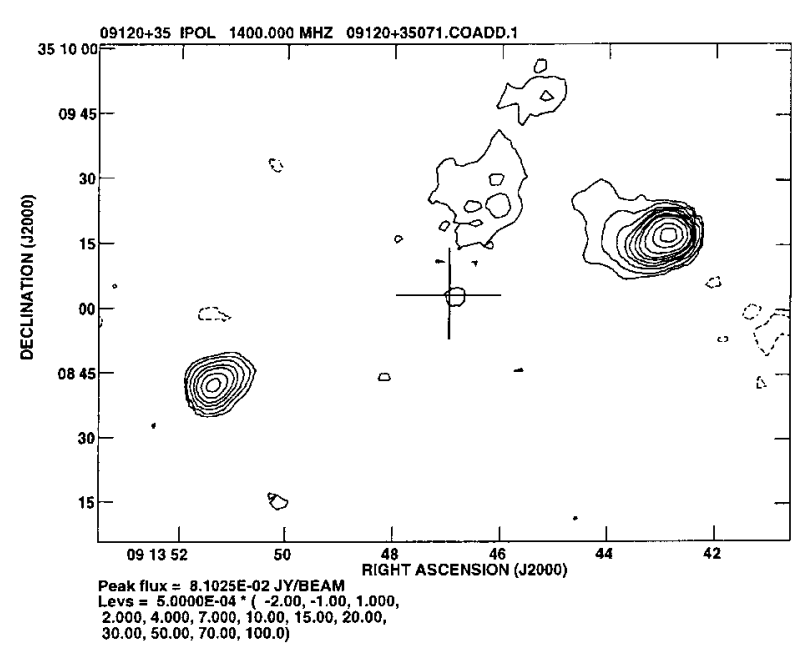

Fig. A2. VLA 1400-MHz contour map of the source $0910+353$ reproduced from the FIRST 09120+35 map (courtesy of R. Becker). FWHB of the restoring beam is $5.4 \times 5.4$ arcsec. The contour levels are are shown under the map. The position of the optical galaxy is marked by the cross

\section{Notes to individual sources in Table 3}

0717+367: in Abell cluster 579

0722+393: Confusing, flat-spectrum, possibly variable source at $07^{\mathrm{h}} 26^{\mathrm{m}} 04^{\mathrm{s}} .8 ;+32^{\circ} 12^{\prime} 28^{\prime \prime}$ ( 5.8 arcmin away). $S_{1.4} \approx 135-210 \mathrm{mJy}$.

0723+488: A featureless optical spectrum was observed in March 1992 with the Asiago 182-cm telescope (cf. Machalski 1992). Variable in the radio (cf. Table 3) and likely in the optical.

0751+485: A featureless optical spectrum was observed in March 1992 with the Asiago 182-cm telescope (cf. Machalski 1992).

0755+379: NGC 2484; UGC 4125. References to WSRT maps and physical parameters in Condon \& Broderick (1988).

0755+480A: Confusing, extended, double source 0755+480B (3.0 arcmin away), identified with a faint galaxy.

0804+499A: 8.4 arcmin apart from very compact variable quasar $0804+499 \mathrm{~B}$.

0804+370: Confusing source at $08^{\mathrm{h}} 08^{\mathrm{m}} 29.44$; $+36^{\circ} 59^{\prime} 43^{\prime \prime} 7, S_{1.4} \approx 60 \mathrm{mJy}$.

$0809+328$ : The radio source is likely associated with one of two close (separation of 2.3 arcsec) galaxies in a pair.

$0810+351$ : Centroid of the double radio source coincides well with the bright elliptical galaxy, however morphological type of the source with evident bright "hot spots" is not typical for galaxies at $z<0.1$.
0816+367: Confusing flat-spectrum source at $08^{\mathrm{h}} 20^{\mathrm{m}} 20.2 ; \quad+36^{\circ} 40^{\prime} 05^{\prime \prime}$ (4.5 arcmin away). $S_{1.4} \approx$ $100 \mathrm{mJy}$.

0818+472: Two confusing sources: $S_{1.4} \approx 190$ mJy at $08^{\mathrm{h}} 21^{\mathrm{m}} 59.0 ;+47^{\circ} 02^{\prime} 53^{\prime \prime}$ (5.2 arcmin away), and $S_{1.4}=54$ mJy, flat-spectrum, at $08^{\mathrm{h}} 22^{\mathrm{m}} 09.6 ;+47^{\circ} 05^{\prime} 53^{\prime \prime}$ (6.9 arcmin away).

0822+394: The spectrum breaks at about $1.4 \mathrm{GHz}$. Low- and high-frequency spectral indices differ significantly: $\alpha_{0.4}^{1.4} \approx 0.42, \alpha_{1.4}^{10} \approx 1.14$.

0829+512: Neither of two suggested galaxy identifications (cf. Paper III) is convincing, however association of the radio source with a faint distant galaxy is very probable.

0838+325: in Abell cluster 694/695. 4885-MHz VLA map of the central part of the source is shown in Fig. A1 (Appendix).

0844+319: IC 2402 galaxy.

0906+480A: Close pair (4.3 arcmin apart) with 0906+480B. Both are very compact; deconvolved size of $0.0 \times 0.0$ arcsec at $5 \mathrm{GHz}$. Spectral indices quoted in Paper IV and Machalski \& Inoue (1990) were erroneously interchanged. Probably variable above $5 \mathrm{GHz}$.

0908+380: Confusing, steep-spectrum source $(0908+380 \mathrm{~B}$ in the B3VLA sample; Vigotti et al. 1989) at $09^{\mathrm{h}} 11^{\mathrm{m}} 48.5 ;+37^{\circ} 50^{\prime} 19^{\prime \prime}$ (3.1 arcmin away). $S_{1.4} \approx$ $150 \mathrm{mJy}$.

0910+353: The VLA A-array map and optical identification of the source published in Paper IV were wrong. The correct map reproduced from the FIRST survey (courtesy of R.H. Becker) with the new identification indicated is shown in Fig. A2 (Appendix).

0911+354: Northern component with $S_{1.4} \approx 50 \mathrm{mJy}$ (cf. VLA A-array map in Paper IV) may be a separate source.

0918+381: Confusing, very steep-spectrum, double source $0919+381$ at $09^{\mathrm{h}} 22^{\mathrm{m}} 15.20 ;+37^{\circ} 54^{\prime} 03^{\prime \prime}$ (6.9 $\operatorname{arcmin}$ away). $S_{1.4}=295 \pm 6 \mathrm{mJy}$.

0927+352: Radio, optical, and X-ray properties of the source are discussed in Machalski \& Brandt (1996).

0928+480: Southern component at $09^{\mathrm{h}} 31^{\mathrm{m}} 34^{\mathrm{s}} .3$; $+47^{\circ} 49^{\prime} 47^{\prime \prime}$ (marked "B" in Paper II) is likely a separate source. $S_{1.4}=186 \mathrm{mJy}$ in NVSS.

0949+354: Confusing, flat-spectrum source at $09^{\mathrm{h}} 52^{\mathrm{m}} 22 \mathrm{~s} .6 ;+35^{\circ} 08^{\prime} 03^{\prime \prime}\left(5.2\right.$ arcmin away). $S_{1.4} \approx$ $50 \mathrm{mJy}$.

0954+346: Confusing, steep-spectrum source at $09^{\mathrm{h}} 57^{\mathrm{m}} 48^{\mathrm{s}} .3 ;+34^{\circ} 17^{\prime} 28^{\prime \prime} \quad(4.8$ arcmin away $) . \quad S_{1.4} \approx$ $90 \mathrm{mJy}$

0955+320: Confusing, steep-spectrum source at $09^{\mathrm{h}} 58^{\mathrm{m}} 25.37 ;+31^{\circ} 47^{\prime} 17^{\prime \prime} 2$ (7.1 arcmin away). $S_{1.4} \approx$ $170 \mathrm{mJy}$

1003+484: $3 \mathrm{C} 235$. The spectrum breaks at about $320 \mathrm{MHz} . \alpha_{0.038}^{0.4} \approx 0.82, \alpha_{0.4}^{15} \approx 1.16$.

1014+397: The optical magnitudes are for the galaxy at $10^{\mathrm{h}} 17^{\mathrm{m}} 18.60 ;+39^{\circ} 31^{\prime} 19^{\prime \prime} 3 \quad$ (cross " 1 " in 
Paper II). Vigotti et al. (1989) identified this extended double source with another brighter galaxy at $10^{\mathrm{h}} 17^{\mathrm{m}} 17^{\mathrm{s}} .86 ;+39^{\circ} 31^{\prime} 46^{\prime \prime} 5$. Thompson et al. (1992) determined it as a NLRG with the redshift of 0.089 .

1017+487: in Abell cluster 1003.

1049+384: The optical spectrum shows intermediate properties between galaxies and quasars (Allington-Smith et al. 1988).

1104+365: The optical spectrum as above.

1127+507: Confusing steep-spectrum source $1126+506$ at $11^{\mathrm{h}} 29^{\mathrm{m}} 12 .^{10} ;+50^{\circ} 23^{\prime} 35^{\prime \prime}$ (6.1 arcmin away). $S_{1.4} \approx 348 \mathrm{mJy}$.

1129+351: The optical spectrum shows intermediate properties between galaxies and quasars (Allington-Smith et al. 1988).

1131+493: UGC 6549; IC 708; in Abell cluster 1314. References to WSRT maps and physical parameters in Condon \& Broderick (1988).

1132+492: IC 711; in Abell cluster 1314. Lowresolution Effelsberg map at $2.7 \mathrm{GHz}$ in Haslam et al. (1978).

1141+466: in Abell clusted 1361. Low-resolution Effelsberg map at $2.7 \mathrm{GHz}$ in Waldhausen et al. (1979). Confusing, flat-spectrum source at $11^{\mathrm{h}} 43^{\mathrm{m}} 10.9$; $+46^{\circ} 23^{\prime} 40^{\prime \prime}$ (5.5 arcmin away). $S_{1.4} \approx 190 \mathrm{mJy}$. For its low-resolution maps cf. Rudnick \& Owen (1977) and Andernach et al. (1980). 4885-MHz VLA map of central part of the source is shown in Fig. A1 (Appendix).

1144+352: A total extent of the radio emission of about 10 arcmin (about $990 \mathrm{kpc}$ ) is suggested from the 4.85-GHz map N111200W of Condon et al. (1989). Its position angle (PA) of about $105 \mathrm{deg}$ is compatible with $\mathrm{PA}=118^{\circ}$ of a two-sided, about $45 \mathrm{kpc}$ extended, emission from the very compact and bright core on the map $11480+35071 \mathrm{~B}$ of Becker et al. (1995). Moreover, the VLBI-scale structure (about 22 mas, corresponding to about $37 \mathrm{pc}$ ) observed by Henstock et al. (1995), has $\mathrm{PA}=121.5$.

1144+497: Confusing source at $11^{\mathrm{h}} 47^{\mathrm{m}} 50.2$; $+49^{\circ} 28^{\prime} 05^{\prime \prime}$ (4.1 arcmin away). $S_{1.4} \approx 80 \mathrm{mJy}$. Though the blue optical object lies on the overall axis of this double source, the identification is not convincing.

1151+384: Bright, confusing, steep-spectrum double source $1151+383$ at $11^{\mathrm{h}} 54^{\mathrm{m}} 01^{\mathrm{s}} .2 ;+38^{\circ} 05^{\prime} 07^{\prime \prime}$ (6.9 $\operatorname{arcmin}$ away). $S_{1.4} \approx 500 \mathrm{mJy}$.

1152+462: Confusing source $1152+463$ at $11^{\mathrm{h}} 54^{\mathrm{m}} 43.95 ;+46^{\circ} 01^{\prime} 32^{\prime \prime} 4$ (7.5 arcmin away). $S_{1.4}=$ $217 \pm 10$ mJy Designated as $1152+462 B$ in Paper V).

1158+345: The optical identification is not certain.

1202+499B: Confusing source 1202+499A (3.0 arcmin away). $S_{1.4}=154 \mathrm{mJy}$.

1216+475: M 106; NGC 4258; UGC 7353. Lowluminosity, starburst galaxy with a trace of non-thermal nucleus. References to WSRT and Effelsberg maps, as well as physical parameters in Condon \& Broderick (1988).
1218+489: Though the blue optical object lies on overall axis of this source (cf. Paper IV), the identification may be an accidental one.

1226+492: Confusing, flat-spectrum source at $12^{\mathrm{h}} 28^{\mathrm{m}} 52^{\mathrm{s}} .9 ;+49^{\circ} 04^{\prime} 36^{\prime \prime}$ (6.6 arcmin away). $S_{1.4} \approx$ $50 \mathrm{mJy}$.

1230+486: Very likely variable at frequencies above $5 \mathrm{GHz}$. Confusing steep-spectrum source at $12^{\mathrm{h}} 32^{\mathrm{m}} 35 \mathrm{~s} .7$; $+48^{\circ} 16^{\prime} 50^{\prime \prime}$ (4.7 arcmin away) identified with $4 \mathrm{C} 48.35$. $S_{1.4} \approx 150 \mathrm{mJy}$.

1231+495: Faint red optical object at $12^{\mathrm{h}} 34^{\mathrm{m}} 20.61$; $+49^{\circ} 14^{\prime} 18^{\prime \prime} 7$ (cf. Paper IV) might be associated with this double radio source.

1237+353: Confusing, compact, steep-spectrum source at $12^{\mathrm{h}} 40^{\mathrm{m}} 19.2$; $+34^{\circ} 56^{\prime} 39^{\prime \prime}$ (6.4 arcmin away). $S_{1.4} \approx 190 \mathrm{mJy}$.

1239+328: NGC 4631; UGC 7865. Low-luminosity star-burst galaxy. References to WSRT, NRAO interferometer, and Effelsberg maps, as well as physical parameters in Condon \& Broderick (1988).

1248+350: Deep observations of the field at $408 \mathrm{MHz}$ (5C12 survey: Benn et al. 1982), at 610 and $1415 \mathrm{MHz}$ (WSRT surveys: Katgert 1978; Katgert et al. 1973, respectively). Identified with 5C 12.2 and $1248+$ $35 \mathrm{~W} 1$. Confusing, faint, double source (5C 12.4; 1248+ $34 \mathrm{~W} 1)$ at $12^{\mathrm{h}} 50^{\mathrm{m}} 46.0 ;+34^{\circ} 39^{\prime} 59^{\prime \prime}$ (4.7 arcmin away). $S_{1.4} \approx 90 \mathrm{mJy}$.

1249+508: $3 \mathrm{C} 277$. The spectrum breaks at about 1.1 GHz. $\alpha_{0.15}^{1.1} \approx 0.90, \alpha_{1.1}^{10} \approx 1.18$.

1256+489: $4 \mathrm{C}$ 48.36. Confusing, steep-spectrum source at $12^{\mathrm{h}} 59^{\mathrm{m}} 42^{\mathrm{s}} .4 ;+48^{\circ} 43^{\prime} 22^{\prime \prime}$ (5.4 arcmin away). $S_{1.4} \approx 110 \mathrm{mJy}$.

1322+366: NGC 5141; UGC 8433. WSRT 5-GHz map in Fanti et al. (1977). Physical parameters in Condon \& Broderick (1988).

1324+498A: Confusing, flat-spectrum source $1324+498 \mathrm{~B}$ at $13^{\mathrm{h}} 26^{\mathrm{m}} 39.3 ;+49^{\circ} 33^{\prime} 55^{\prime \prime}$ (4.1 arcmin away). $S_{1.4} \approx 90 \mathrm{mJy}$.

1327+474: M 51; NGC 5194; UGC 8493. Lowluminosity star-burst galaxy with faint non-thermal radio core. References to WSRT and Effelsberg maps, as well as physical parameters in Condon \& Broderick (1988). The spectrum breaks at about $1.4 \mathrm{GHz} . \alpha_{0.038}^{1.4} \approx 0.67$, $\alpha_{1.4}^{5} \approx 1.07$.

1329+503: Confusing, steep-spectrum source at $13^{\mathrm{h}} 30^{\mathrm{m}} 56.4 ;+50^{\circ} 09^{\prime} 04^{\prime \prime} \quad(6.7$ arcmin away $) . S_{1.4} \approx$ $200 \mathrm{mJy}$.

1348+352: Unique radio source; a more detailed discussion in the Appendix.

1354+325: For a discussion of the optical field, cf. Machalski \& Condon (1985b).

1413+349: Confusing, steep-spectrum source at $14^{\mathrm{h}} 16^{\mathrm{m}} 17^{\mathrm{s}} \cdot 2 ;+34^{\circ} 48^{\prime} 05^{\prime \prime} \quad\left(4.4\right.$ arcmin away). $S_{1.4} \approx$ $130 \mathrm{mJy}$. 
1415+463: 4C46.29. Confusing source 1414+463 at $14^{\mathrm{h}} 16^{\mathrm{m}} 42^{\mathrm{s}} .9 ;+46^{\circ} 03^{\prime} 04^{\prime \prime}(6.0$ arcmin away $) . S_{1.4} \approx$ $140 \mathrm{mJy}$.

1452+502A: Close pair (2.5 arcmin apart) with compact quasar $1452+502 \mathrm{~B}$. All flux densities in the surveys given in Col. 2 are strongly confused.

1504+346: in Abell cluster 2025.

1507+476: The optical galaxy is 2.1 arcsec apart from this compact radio source whose observed structure was deconvolved into two "point" components separated by 0.87 arcsec (Paper III).

1527+349: For a discussion of the optical field cf. Paper III.

1546+487: Confusing, steep-spectrum source at $15^{\mathrm{h}} 47^{\mathrm{m}} 40^{\mathrm{s}} .4 ;+48^{\circ} 39^{\prime} 22^{\prime \prime} \quad\left(7.1\right.$ arcmin away). $S_{1.4} \approx$ $220 \mathrm{mJy}$.

1613+345: Two confusing sources at $16^{\mathrm{h}} 15^{\mathrm{m}} 39.2$; $+34^{\circ} 21^{\prime} 57^{\prime \prime}$ and $16^{\mathrm{h}} 15^{\mathrm{m}} 44^{\mathrm{s}} .5 ;+34^{\circ} 21^{\prime} 15^{\prime \prime}$ (4.8 and 6.1 arcmin away, respectively), was recorded as $1613+344$ in Owen et al. (1983) with a total flux $S_{1.4} \approx 70 \mathrm{mJy}$.

1615+351: NGC 6109; UGC 10316. References to WSRT maps, and physical parameters in Condon \& Broderick (1988).

1626+396: NGC 6166; UGC 10409. WSRT 1.4 and $5 \mathrm{GHz}$ maps in Jaffe \& Perola (1974). Physical parameters in Condon \& Broderick (1988).

1636+379: in Abell cluster 2214. 
Table 3. The revised GB/GB2 sample

总

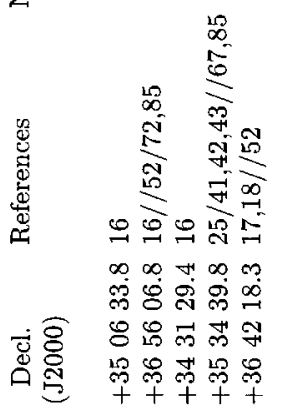

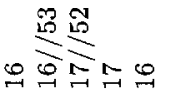

虽觜

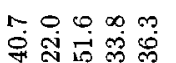
8 츤

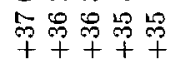
$\infty \stackrel{\infty}{\infty}$ 요용

०ิ \&8요유

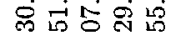
드슨 등ํํㅇํㅇำ

๓

番惡

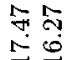

战

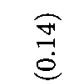

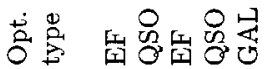

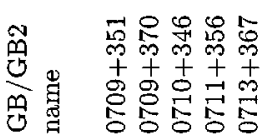

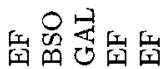

종 还

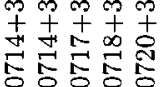

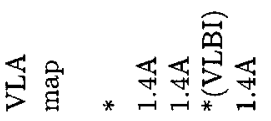

乐谋

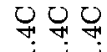

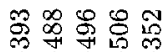

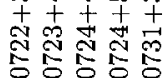

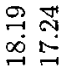

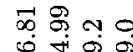

总盆哭

é巳

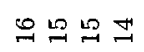

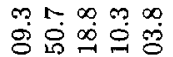

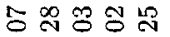

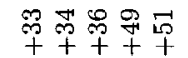

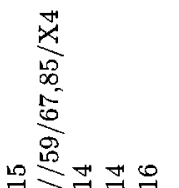

स 15000

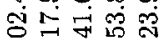

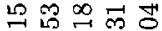

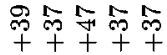
요 $87 \%$

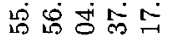
品品命占承 동동혀융웅

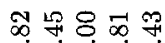

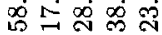

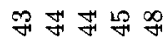

능영송영

赵

$\stackrel{8}{\circ}$

๙ิ

กั่

$\underset{8}{\stackrel{8}{\circ}}$

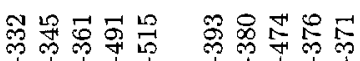

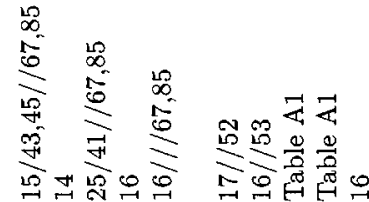

0 is $\infty 00$

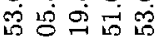

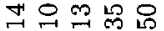

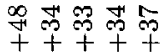

Tr. 0.0

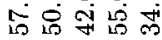

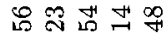

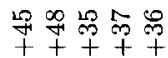

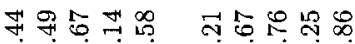

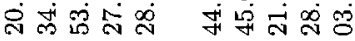

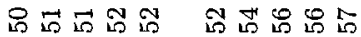

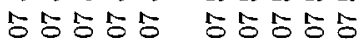

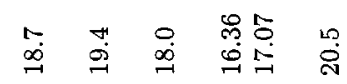

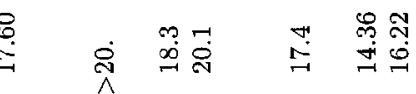

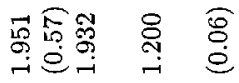

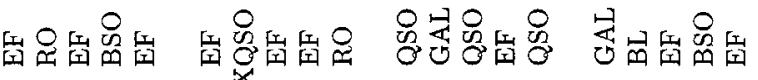

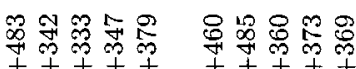

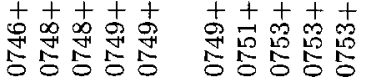
鱼 舀

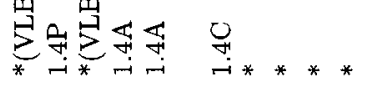

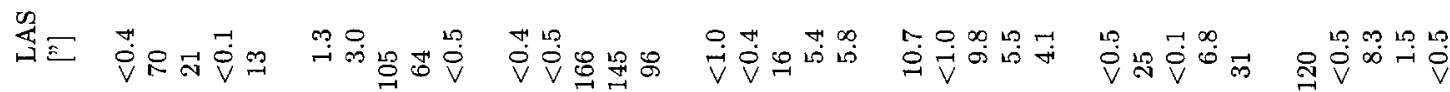

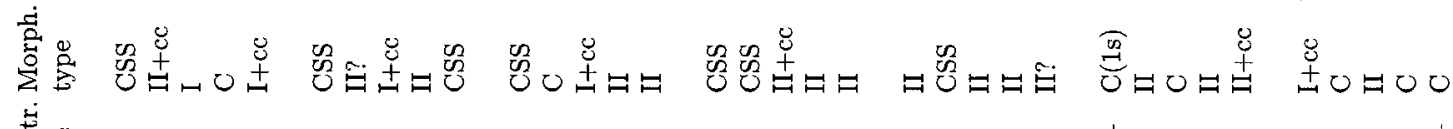

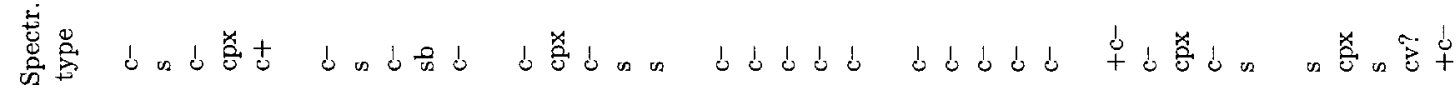
营运

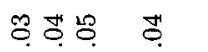

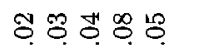

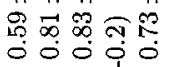
$+H+H+H \quad H+H$ จํ.

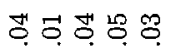
웅 \%웅 $H+H+H$

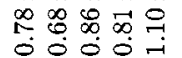
$H+H H+$ $H+H+$ จ $\approx$ ธ Oㅇㅇㅇㅇㅛ

采。品士

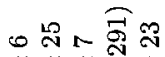

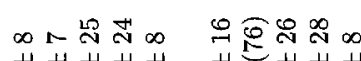

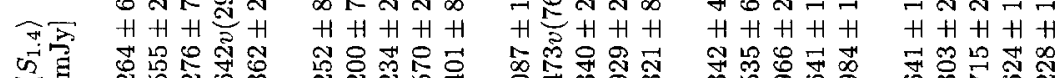

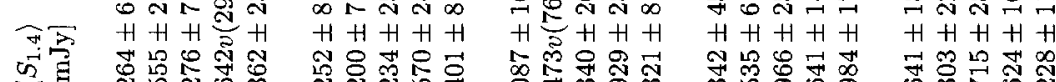

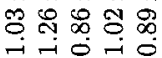

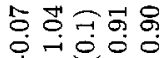
H H H $\stackrel{0}{0}: 0.0$

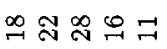
으옹ㅎㅇㅇㅠ

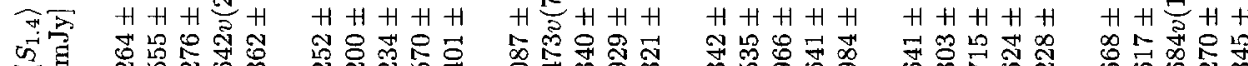

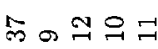

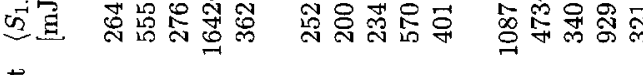
究 mid

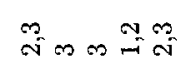

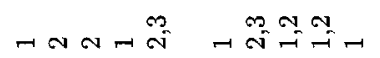

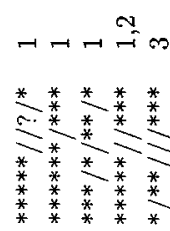
Ir-
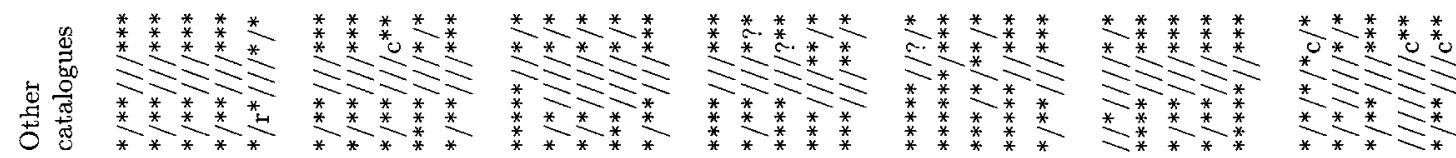

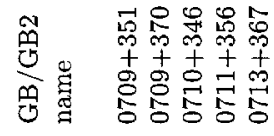

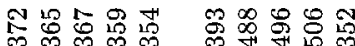

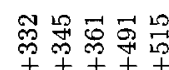

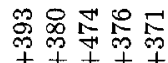

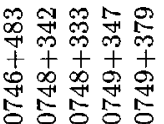

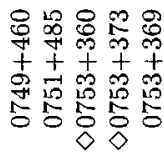


Table 3. continued

\begin{tabular}{|c|c|c|c|c|c|c|c|}
\hline & & $+t$ & & + & + & + & \\
\hline$\theta_{1} \Rightarrow$ & 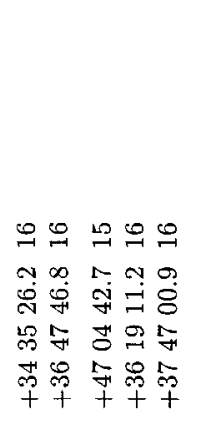 & 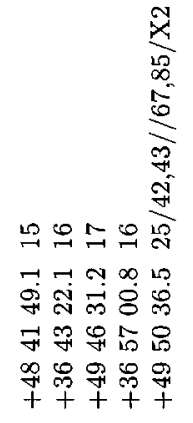 & 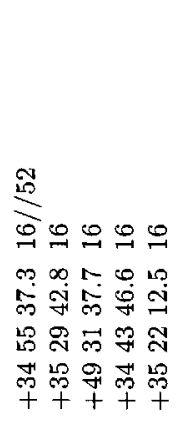 & 变落 & 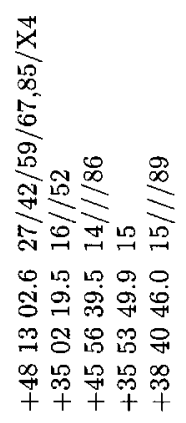 & 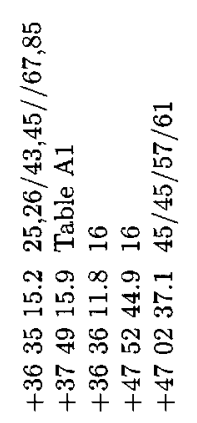 & 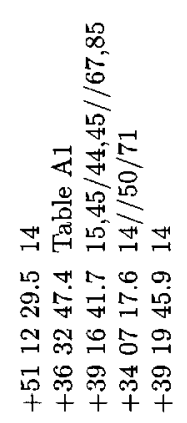 \\
\hline & 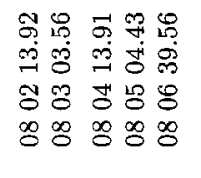 & 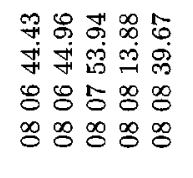 & 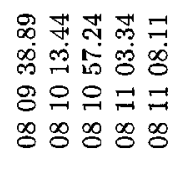 & $\infty$ & 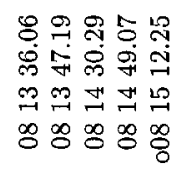 & $\stackrel{\infty}{\circ} \stackrel{0}{\circ} \stackrel{0}{0} \stackrel{0}{0} 0$ & \\
\hline & 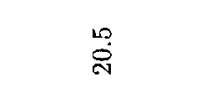 & 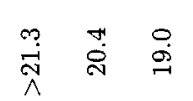 & $\begin{array}{l}\infty \\
\infty \\
\stackrel{\leftrightarrow}{-1} \\
\text {. }\end{array}$ & 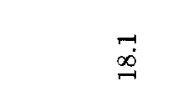 & 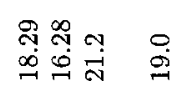 & 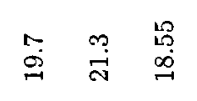 & $\stackrel{\infty}{\stackrel{\leftrightarrow}{\prime}}$ \\
\hline & $\stackrel{\infty}{\infty}$ & $\stackrel{\infty}{\stackrel{\infty}{\infty}} \vec{\infty}$ & 总 & 赵赵赵 & 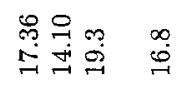 & 吾 & 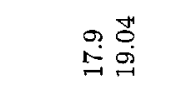 \\
\hline & & 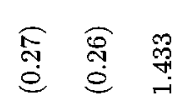 & $\begin{array}{l}\hat{0} \\
\stackrel{1}{\ominus}\end{array}$ & $\stackrel{\text { 突 }}{\ominus}$ & 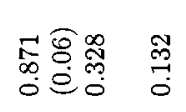 & $\stackrel{\text { ڤ̆ }}{\text { : }}$ & ๙઼! \\
\hline & 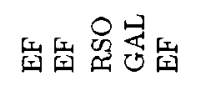 & 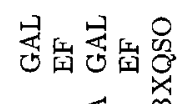 & 哲 & 鼠至盖品 & 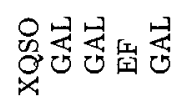 & 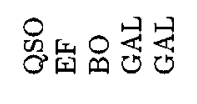 & 至 \\
\hline & 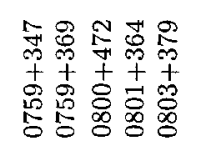 & 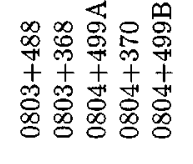 & 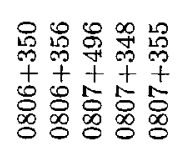 & 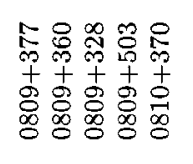 & 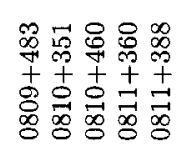 & 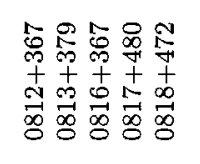 & $\begin{array}{l}+1 \\
0 \\
0 \\
\infty \\
0 \\
0 \\
0\end{array}$ \\
\hline & 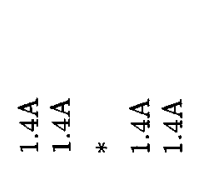 & $\stackrel{\mathbb{4}}{-}$ & 驻出要出 & 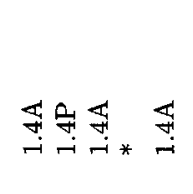 & 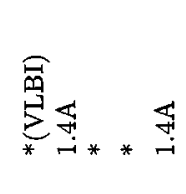 & 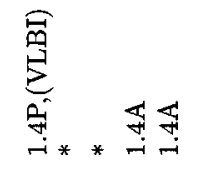 & 畣 \\
\hline & 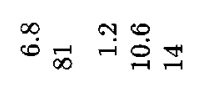 & 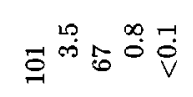 & $\exists \stackrel{\circ}{i} \mathbb{N}$ 的品 & & $\mathscr{L}$ & 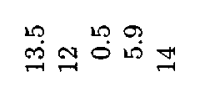 & $\vec{v}$ \\
\hline & 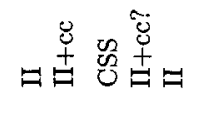 & 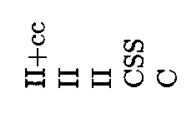 & 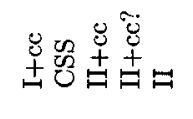 & ヨココロ音 & 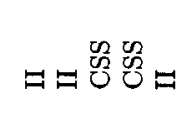 & 预 & - \\
\hline & $\begin{array}{llll}1 & 0 & 0 & 0\end{array}$ & \pm & $t \infty$ & ¿ d d d d & & b & \\
\hline & 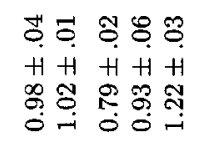 & 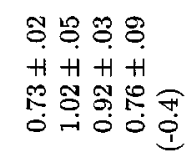 & $\begin{array}{l}+0 \\
\dot{H} \\
\dot{7} \\
0\end{array}$ & 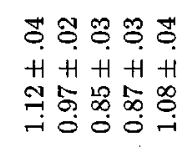 & & + & 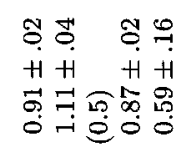 \\
\hline 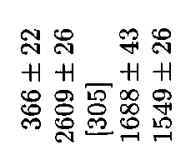 & 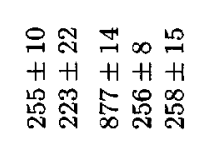 & 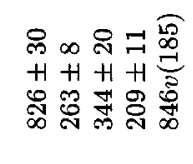 & 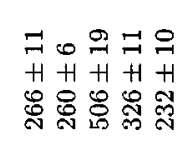 & 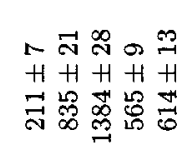 & 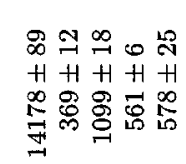 & 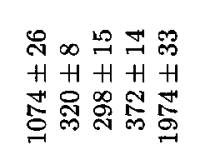 & 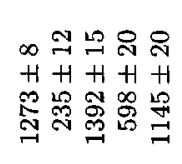 \\
\hline & 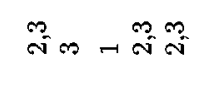 & 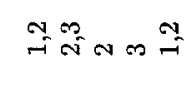 & 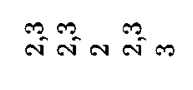 & ๓ & $-\stackrel{\infty}{i}-\underset{\sim}{\sim}-$ & 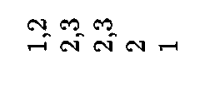 & $\rightarrow \sigma$ \\
\hline & $*$ & 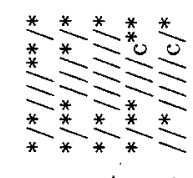 & * & $\frac{*}{*}$ & & & \\
\hline 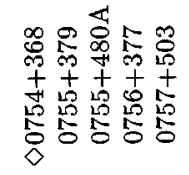 & 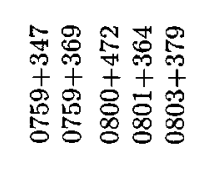 & 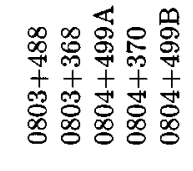 & 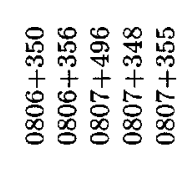 & 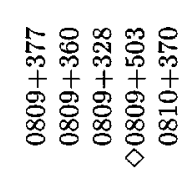 & 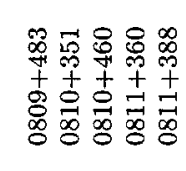 & 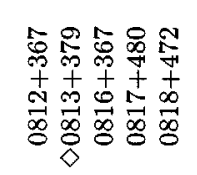 & 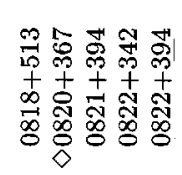 \\
\hline
\end{tabular}


Table 3. continued

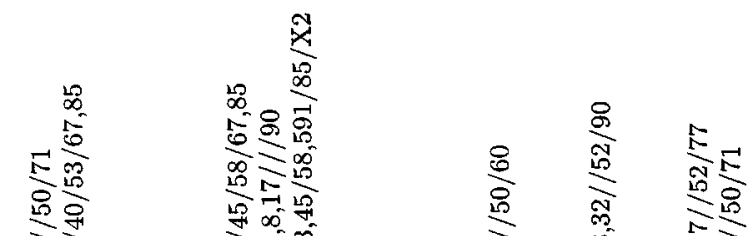

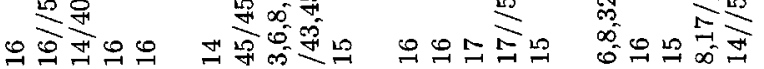

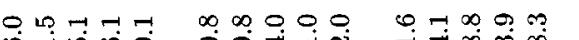

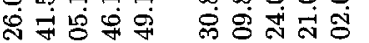

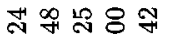

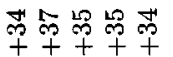
ำ 고 오용

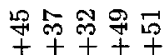

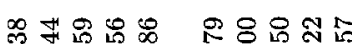

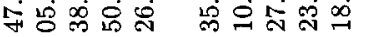

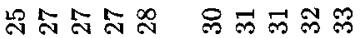
$\stackrel{\infty}{\circ} \stackrel{\infty}{\circ}: \infty$

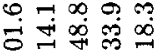

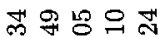

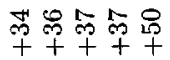

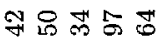

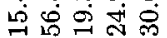
比占我品品 용

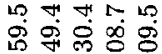

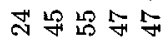

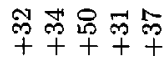

ㄷำ

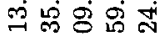

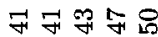

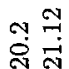

I

冚

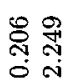

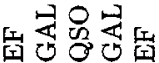

लि

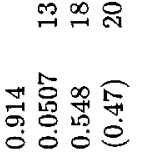

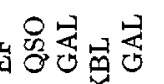

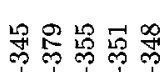

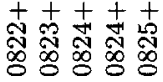

$\infty \stackrel{\circ}{\circ} \%$

(n)

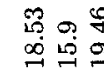

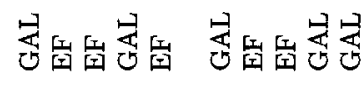

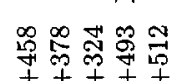

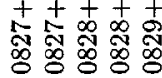

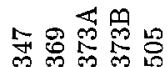

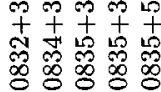

응 $\infty$

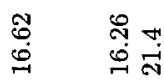

管

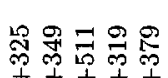

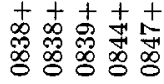

$\stackrel{8}{\square}$

$$
\text { (항 }
$$

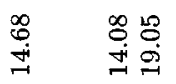

政

留品留留昰

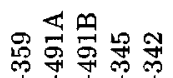

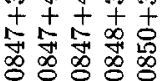

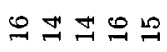

占

눈요용요

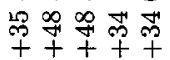

운규용늠

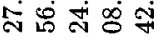

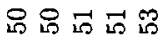

용 용 용

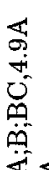

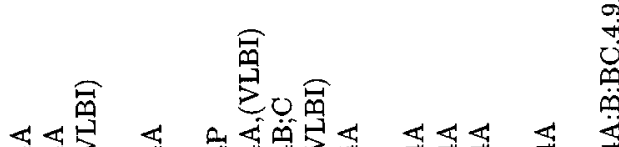

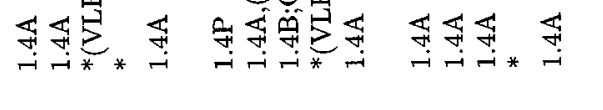

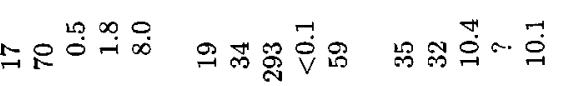

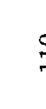

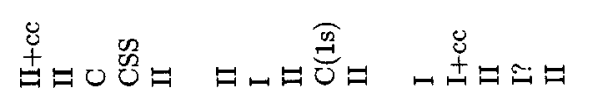

$$
\text { b }
$$

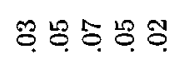

H H H H

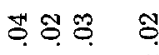

중쭈음쭈용

त्ञ

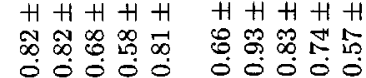

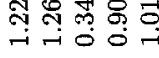

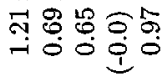

政

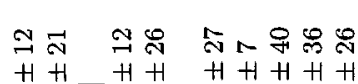

$9 \infty_{\infty}=$

$+H+1+4+1$

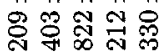

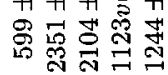

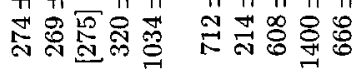

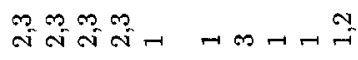

m

- - - 근
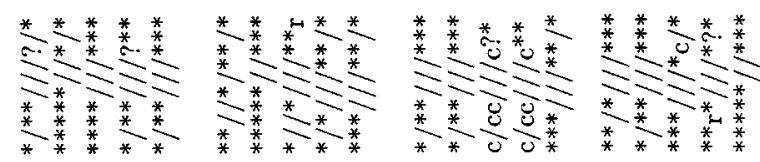

ช่ $\stackrel{4}{9}$

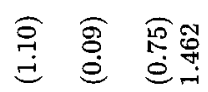

橤

골

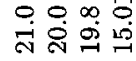

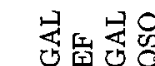

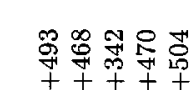

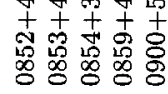

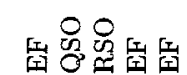

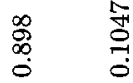

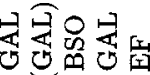

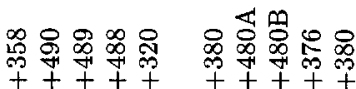

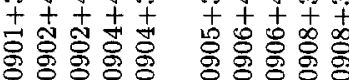

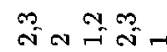

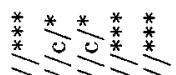

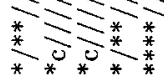

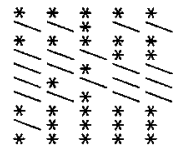

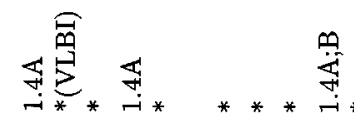

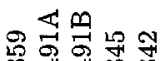

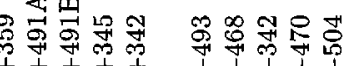

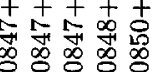

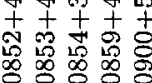

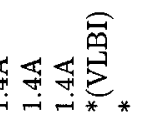

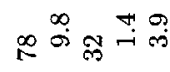

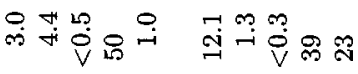

$\underset{8}{8}$

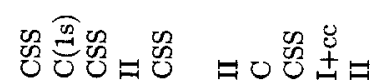

on $\frac{1}{2}$ d。

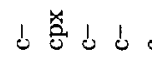

d. $\infty$ d d

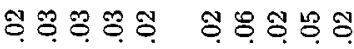

$+\mathrm{H}+\mathrm{H}+\mathrm{H}$

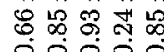

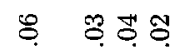

ㅍㅇㅇㅇㅇ 용 छㅇ

H H H H

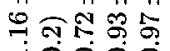

$H+H+H+H$

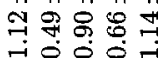

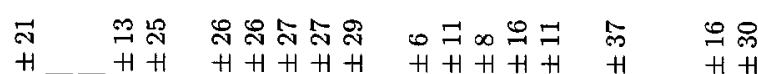

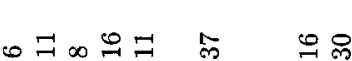

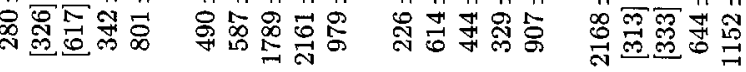

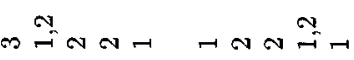
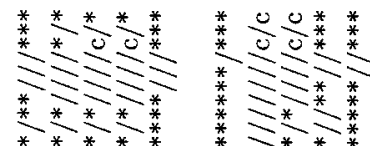

$* * * *$

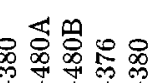

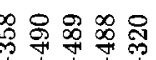

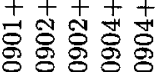

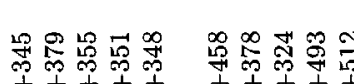

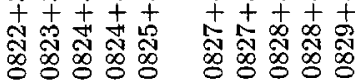

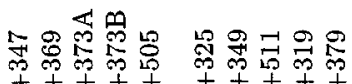

声卉南南 
Table 3. continued

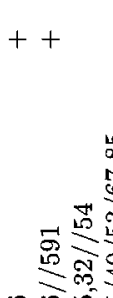

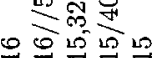

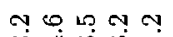

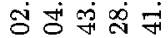

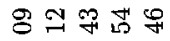

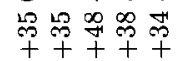

욕가유용

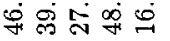

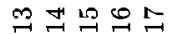

8888

芯 令

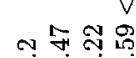

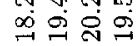

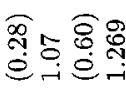

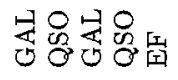

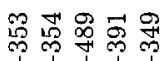

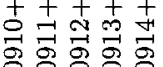

$+\sqrt{n}$

5

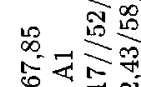
엉

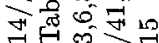

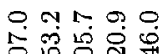

보용

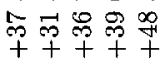

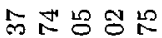

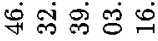

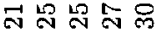

용ㅇㅇㅇㅇㅇ

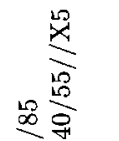

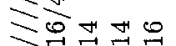
$\infty 0.0 \%$ 성 它影证的品

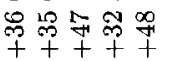

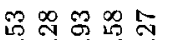

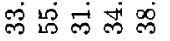

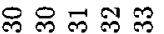
용용 8

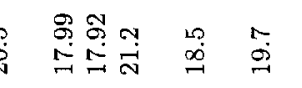$$
\text { : }
$$$$
\text { 吕 }
$$

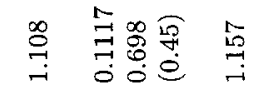

$\stackrel{5}{\stackrel{5}{\longrightarrow}}$

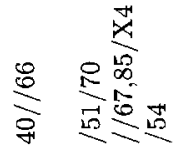

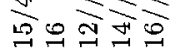

N N N 0

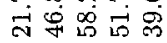

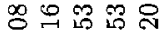
早芦哭学市

$0 \infty \mathbb{\infty}$

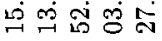

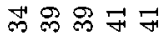
용용용 $\stackrel{\infty}{\text { 郘 }}$

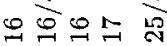

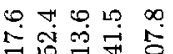
กึำ ำำ

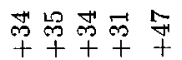
$\infty 8$ मू

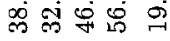

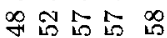
9888

ڤ.

妾迸是

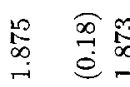

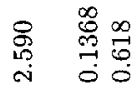

骂总品出出

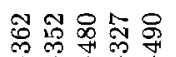

동쇼용ㅇㅇㅇ

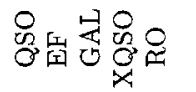

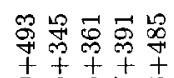

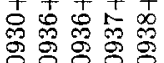

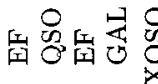

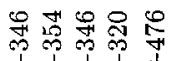

茫

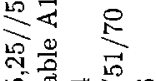
点 ๙ึ

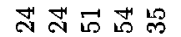

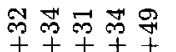

8 옹

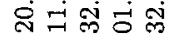

品 50

용으으음으

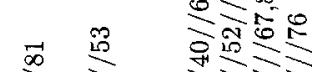

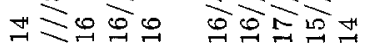

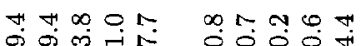

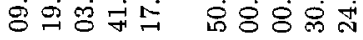

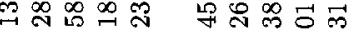

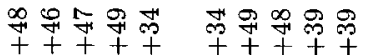

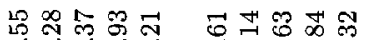

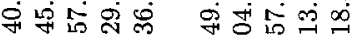

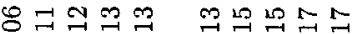

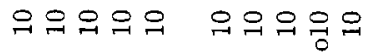

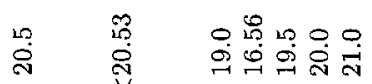
号

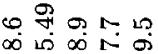

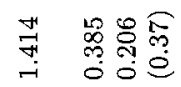

$\stackrel{+}{i}$

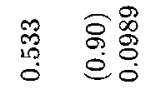

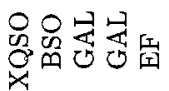

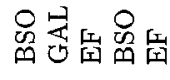

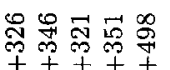

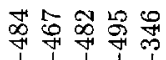

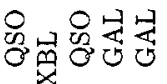

菑

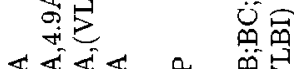

岑岑热

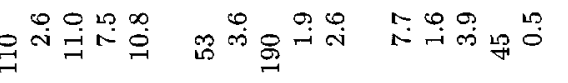

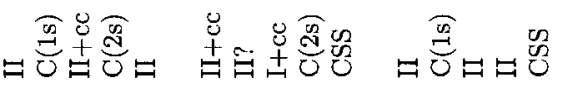

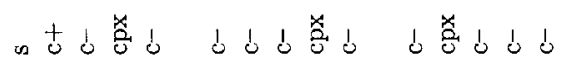

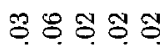

$+\dot{H}+\dot{H}+H$

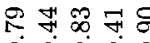

8 $\% 5$

$+\mathrm{H}+\mathrm{H}$

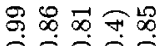

- 00 o

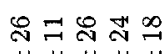

$\mathrm{H}+\mathrm{H} H \mathrm{H}$

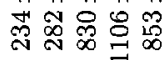

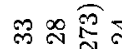

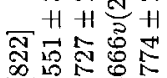

H

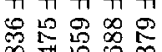

๓

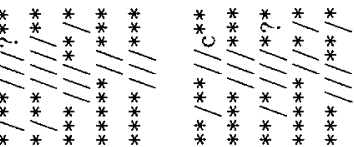

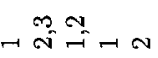

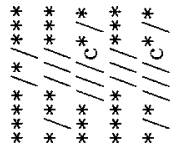

(a)

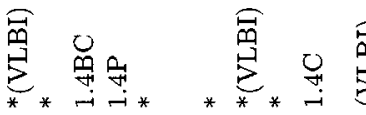

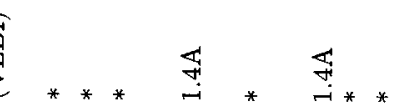

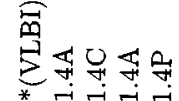

ఫั่

芦嵒亩兽 \&

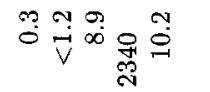

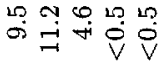

붕유용

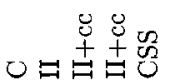

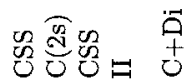

○Oヨ妾

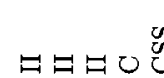

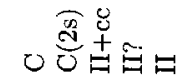

응

$1 \stackrel{1}{1} \stackrel{1}{t}$

릉

- $\mathfrak{3}$

†⿺尢丶

50

$+\mathrm{H}+\mathrm{H}+\mathrm{H}+\mathrm{H}$

舟58

$+\mathrm{H}+\mathrm{H}+\mathrm{H}$

サ $8 \% 5$

H $+\mathrm{H}+$

Бा $\approx 5 \%$

$+\mathrm{H}+\mathrm{H}+\mathrm{H}$

응후웅

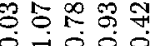

용뭉요

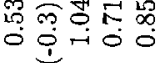

ำ

$\mathrm{H}+\mathrm{H}+\mathrm{H} H$

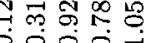

쿠 $\stackrel{\infty}{\forall} \stackrel{9}{\rightarrow}$

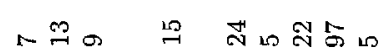

요

폭유용요

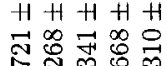

HH+

$\mathrm{H} H \mathrm{H} H \mathrm{H}$

$H+H+H$

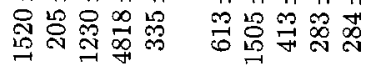

$\mathrm{H}+\mathrm{H}+\mathrm{H}+\mathrm{H}$ N $\infty$ की की

$n \rightarrow-\infty$

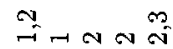

กิ ก लr

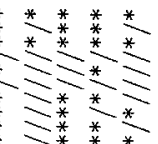

$\stackrel{*}{*}^{*} \stackrel{*}{*}^{*} \stackrel{*}{*}^{*}$

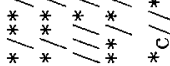

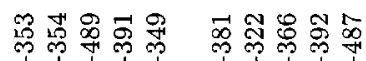

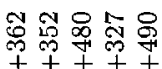

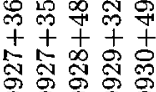

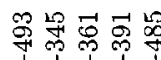

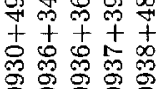

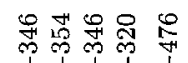

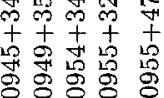

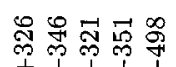

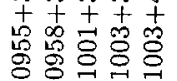
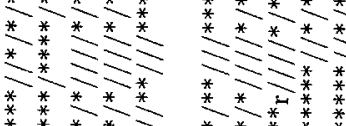

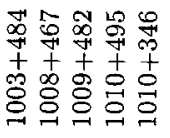
古吉志志声吉

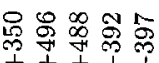


Table 3. continued

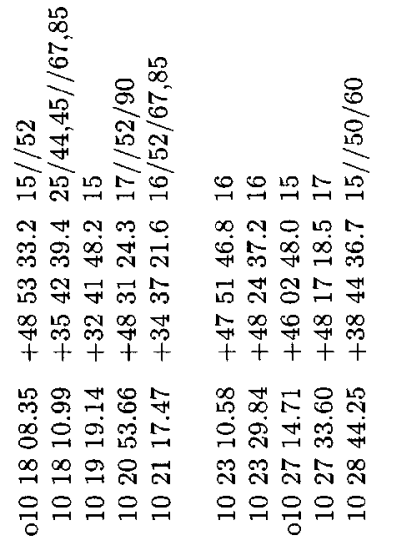

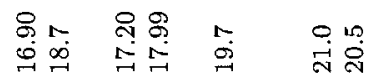

永

केष्ञ

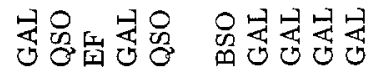

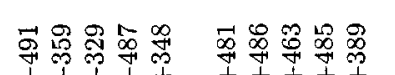

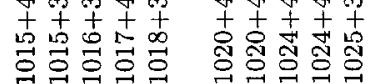

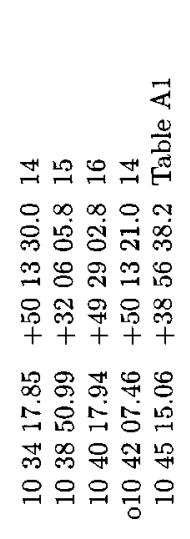

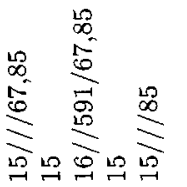

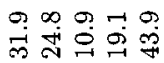

농

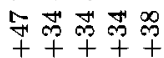

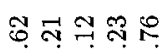

के

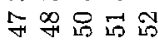

으으응요욤

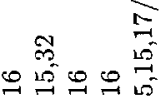

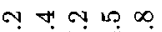

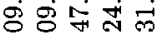

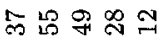

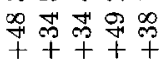

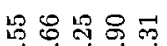

$\pi$ 車宓灾

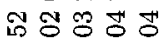

욬ㅋㅍ
号

员点 勇是

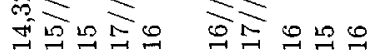

त 000 म $000 \%$

ज的的品

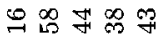

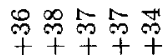

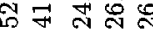

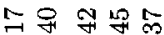

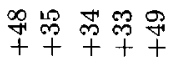

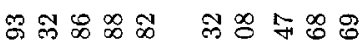

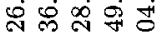

동용요음

ㅋㅋㅋㅋㄱㅎㅁ

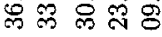

으년용

$\exists \vec{z} \exists \exists \exists$

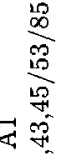

감

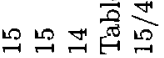

- 0 \%

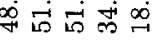

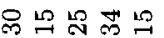

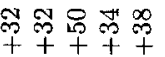

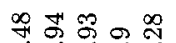

엉워웡

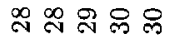

$\exists \Xi \Xi \Xi \Xi$

$\infty$

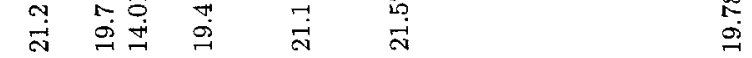

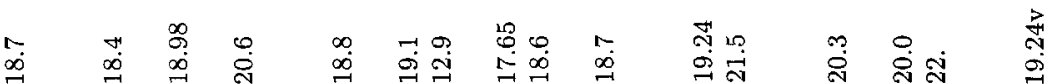

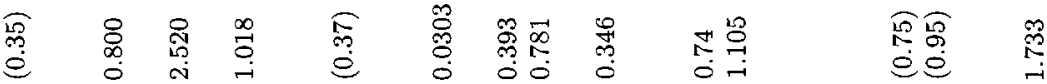

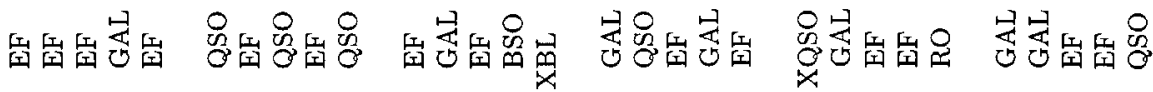

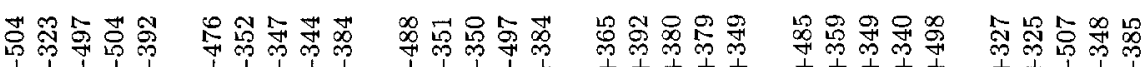

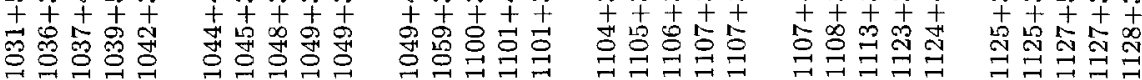

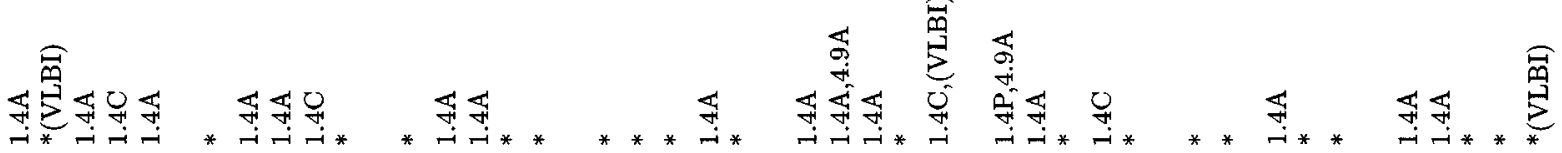

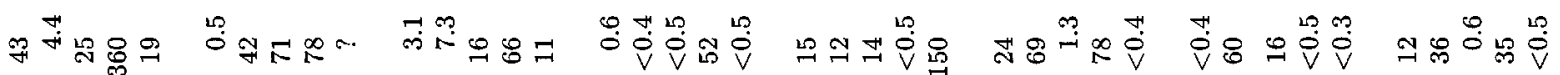

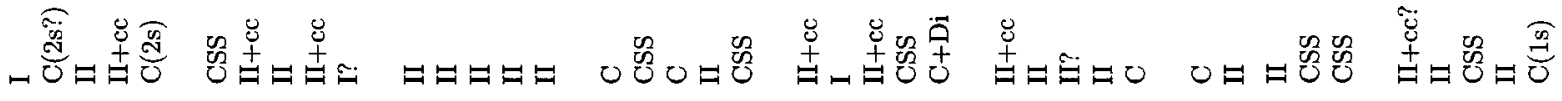

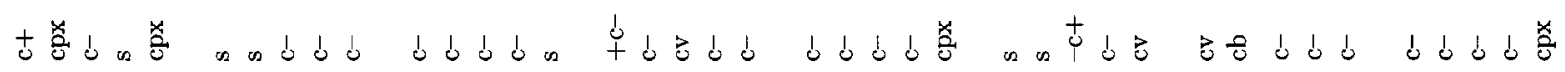

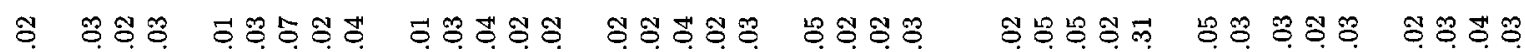

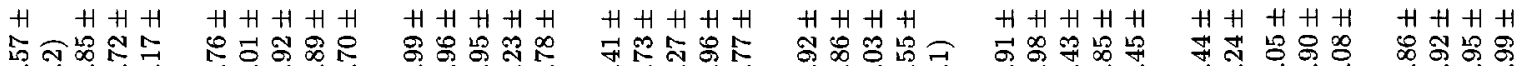

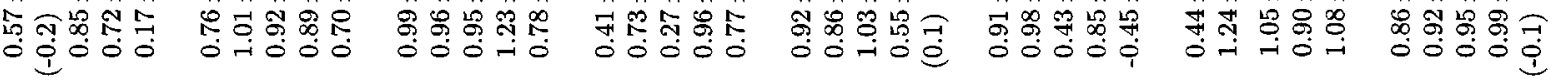

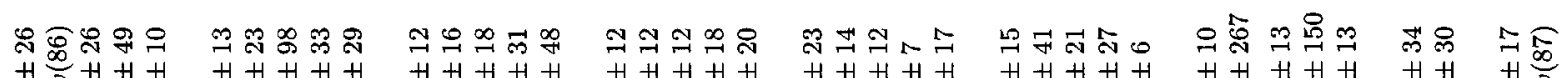

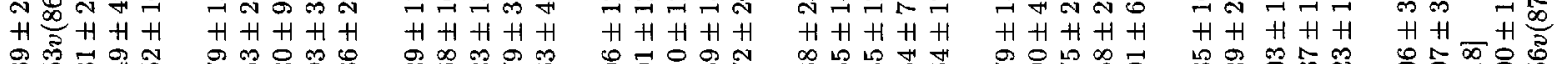

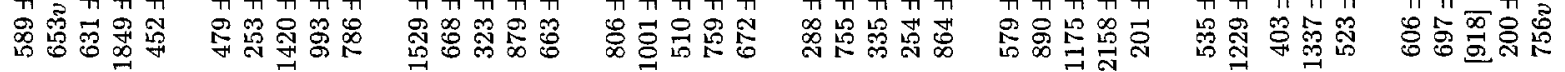
그대

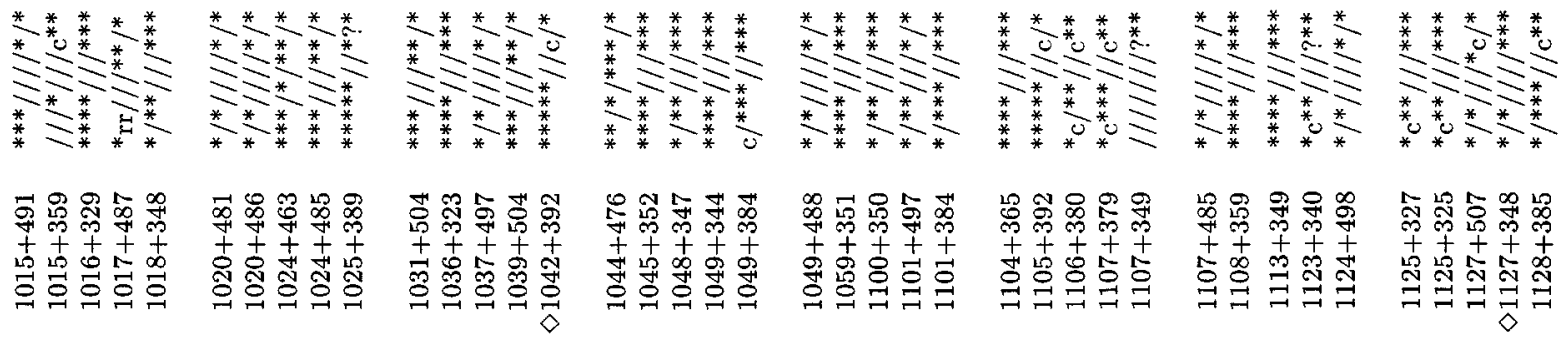


Table 3. continued

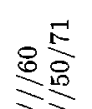

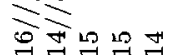

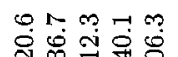

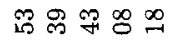

芦芦突吊古

象芯吅 을

लें

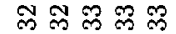

$\exists \vec{\exists} \exists \rightrightarrows \exists$

ํำ

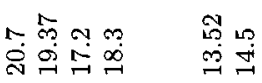

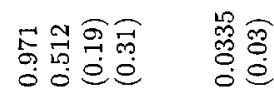

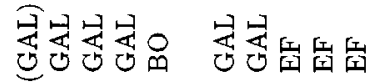

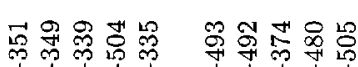

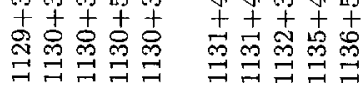

\section{8

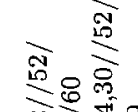

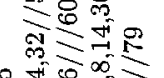

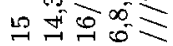

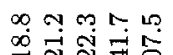

的 $\vec{N} \infty$

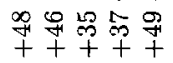

두으요요

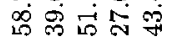

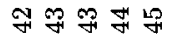

킴ㅋㅋ

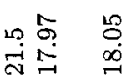

○。

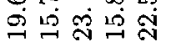

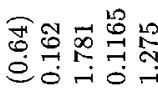

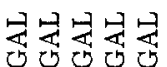

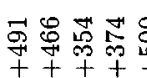

亭声寻声等

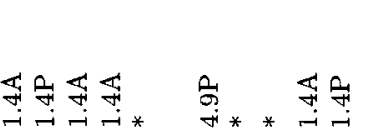
要
$++$

芯

กิ

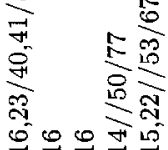

L

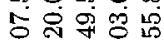

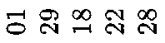

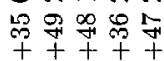

ఇ 듀

สำ ㅇำ

年条命䠉

$\exists \rightrightarrows \exists \rightrightarrows F_{0}$

䒠。

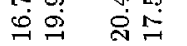

8 \% 아요

㝏

$\stackrel{\infty}{\rightarrow}$

尽

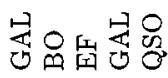

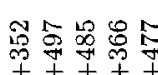

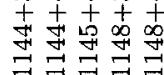

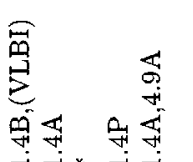

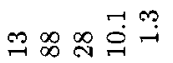

$\stackrel{\circ}{\circ} \stackrel{\infty}{\circ}=$ 命

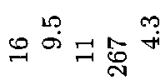

ㅊํㅇํㅇㅇㅛ

日コ产草界

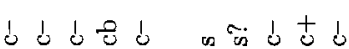

ㅇํㅇํㅇํํㅇํㅇㅇํㅇํㅇ

$H+H+H \quad H+H+H+H$

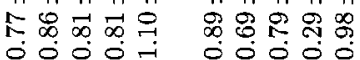

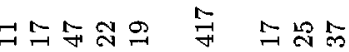

$H H H+H \quad H H H$

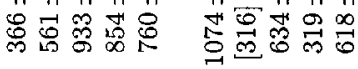

คูก กี-

กัN

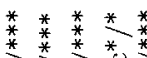

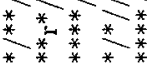

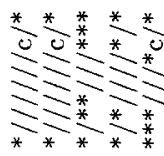

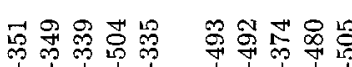

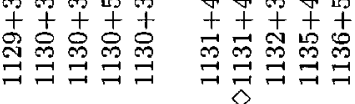

ชุ

$\mathrm{H}+\mathrm{H}+\mathrm{H}+$

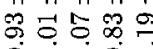

$0-i-10$

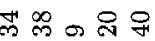

$\mathrm{H}+\mathrm{H}+\mathrm{H}$

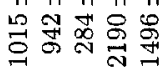

$\stackrel{\Re}{\sim} \rightarrow-$

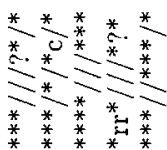

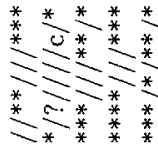

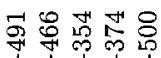

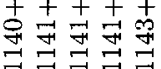

$\mathrm{H}+\mathrm{H}+$

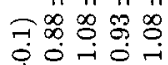

요요

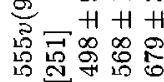

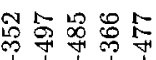

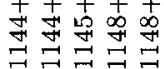

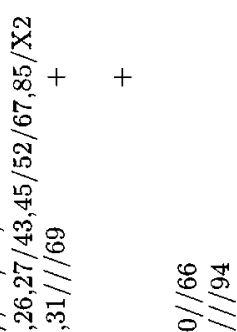

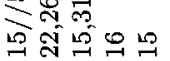

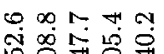

เู

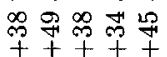

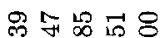

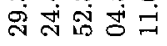

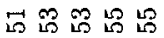

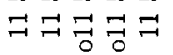

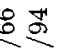

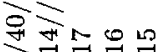

ศ เอ ศบ ॠ म

ตั่ $\stackrel{\infty}{\rightarrow}$

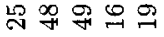

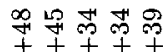

수응용

ते लों

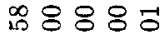

$\exists \stackrel{ }{ } \stackrel{ }{ }$

ำํำำ 10

슨

$\stackrel{\circ}{\stackrel{9}{\rightarrow}}$

80
0
0

ㄴำ논

ลี่

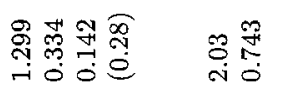

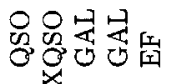

只安究岳

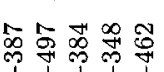

0
$\infty$
10
+
+

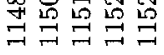

穿

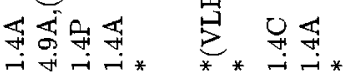

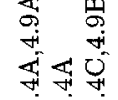

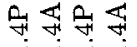

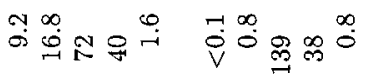

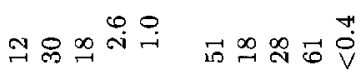

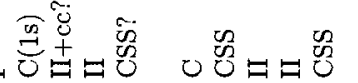

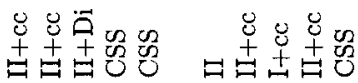

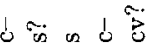

롱

$+\downarrow$

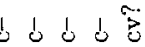

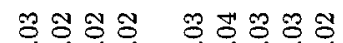

$\dot{H}+\dot{H}+\dot{H}$

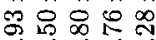

ஜ ず ஜ

$\mathrm{H} H \mathrm{H}+$

Эิ50

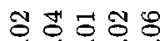

$\mathrm{HH}+\mathrm{H}+\mathrm{H}$

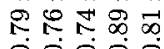

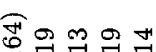

우용교 격

$\mathrm{H}+\mathrm{H}-\mathrm{H}$

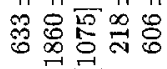

计 H H

$\infty \quad \infty$

H N

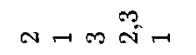

$\sin n a n$
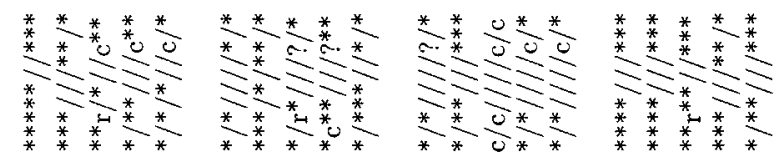

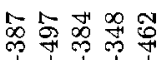

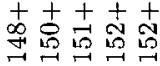

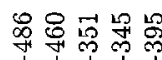

虽志古古古

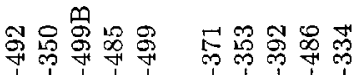

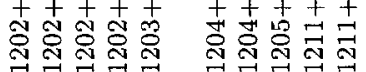


Table 3. continued

等

Cกำ

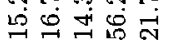

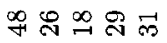

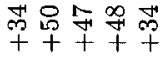

음ํํํำ 귀ำ

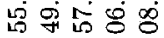

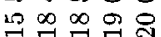

ㄱำ ㄱำ ำ ำ

용

ถ $\infty$ ก

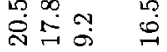

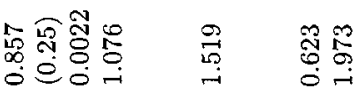

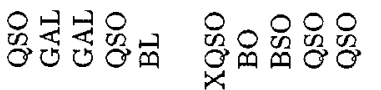

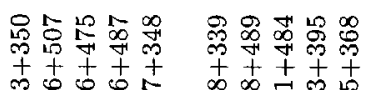

$\frac{2}{9}=\frac{1}{\infty}$

舟里照过

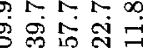

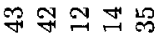

艄市界

$\infty \bar{\infty} \infty \stackrel{\infty}{\infty}$

용요

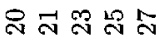

곡코곡고 곡 $++\quad++$

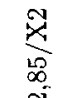

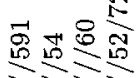

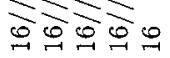

ก 0.0 ㄴ.

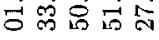

品式呇品

索芦吉导

쇼

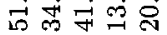

少 ल

코고쿼곡

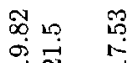

品 趾

穴

营

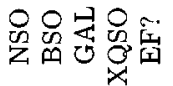

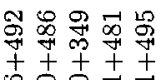

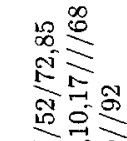
1200 음 ल

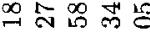

낵요용 중

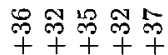

우우요 후

용

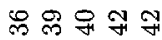

국오곡곡 구

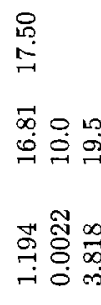

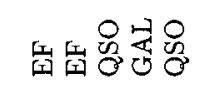

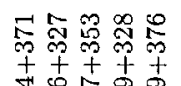

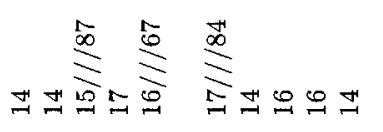

개 구 둔

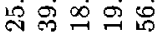

앙 교

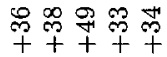

오늠ำ

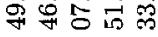

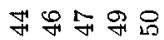

ㄱํㄱำㄱํㅇ 그

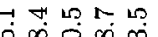

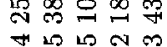

员年茄品命

아우궁

ซึ心

돈ำ

고곡곡

苍 穴芒苍

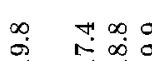

-

点命灾

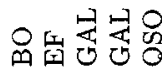

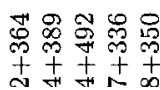

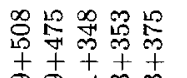

$\stackrel{2}{\frac{2}{0}}$

츤드는

수 0 . $\infty$

穴

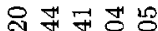

눤

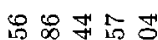

占羿开密京

识战路8 8

곡고고곡

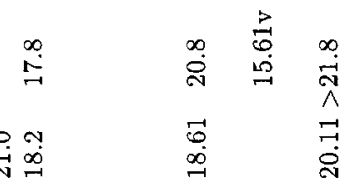

范

尽

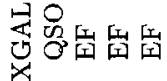

星留崫崖

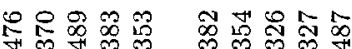

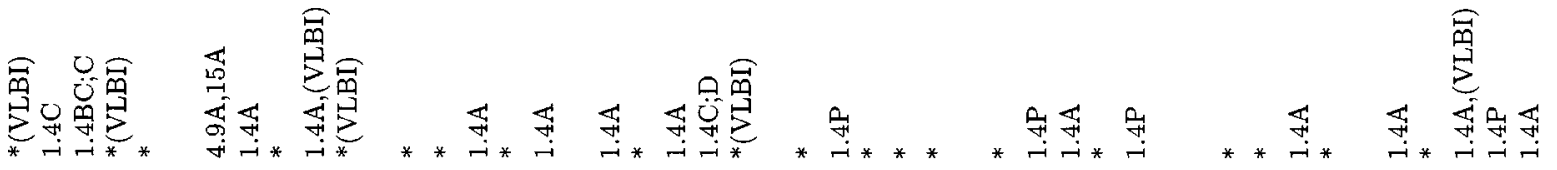

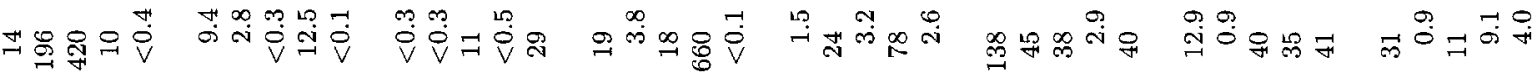

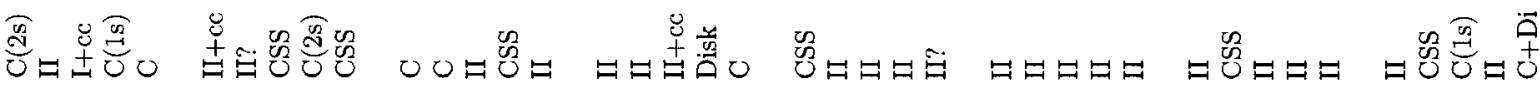

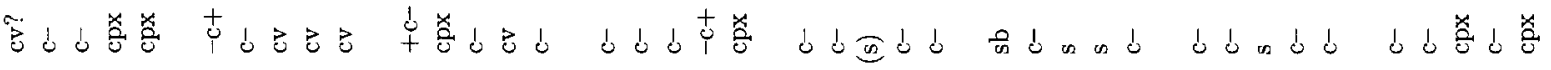

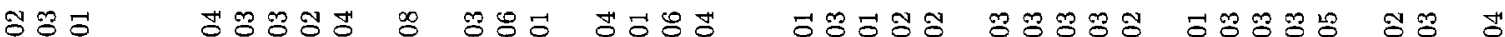

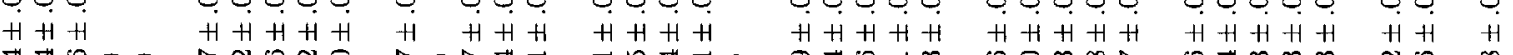

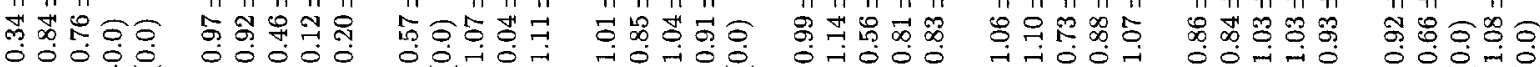

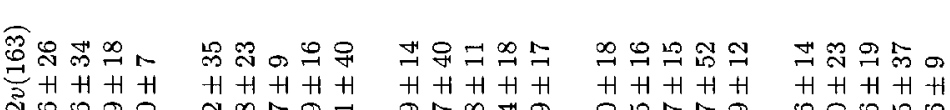

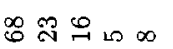

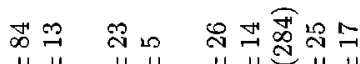

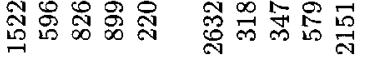

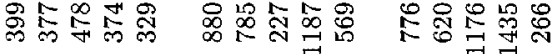

$\rightarrow+1+H$

$H+H+H$

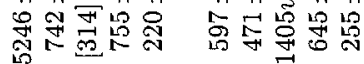

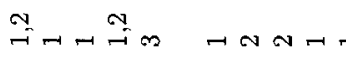

$\cos \pi x$

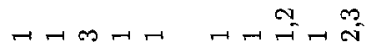

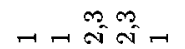

$t \rightarrow a t m$

$\rightarrow \stackrel{4}{4} \rightarrow+4$

* * * * * * *

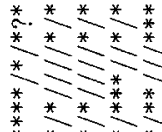

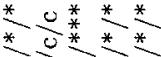

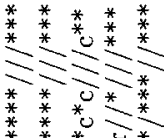

$\stackrel{\underbrace{*}}{*}$
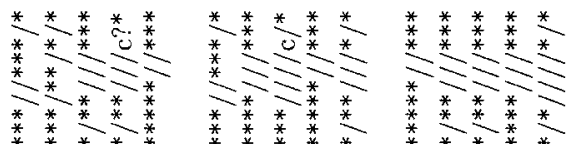

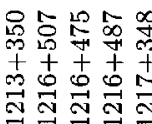

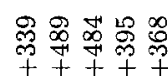

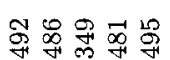

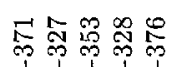

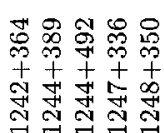

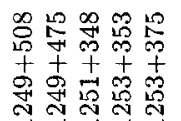

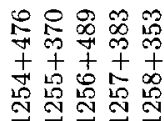

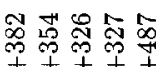


Table 3. continued

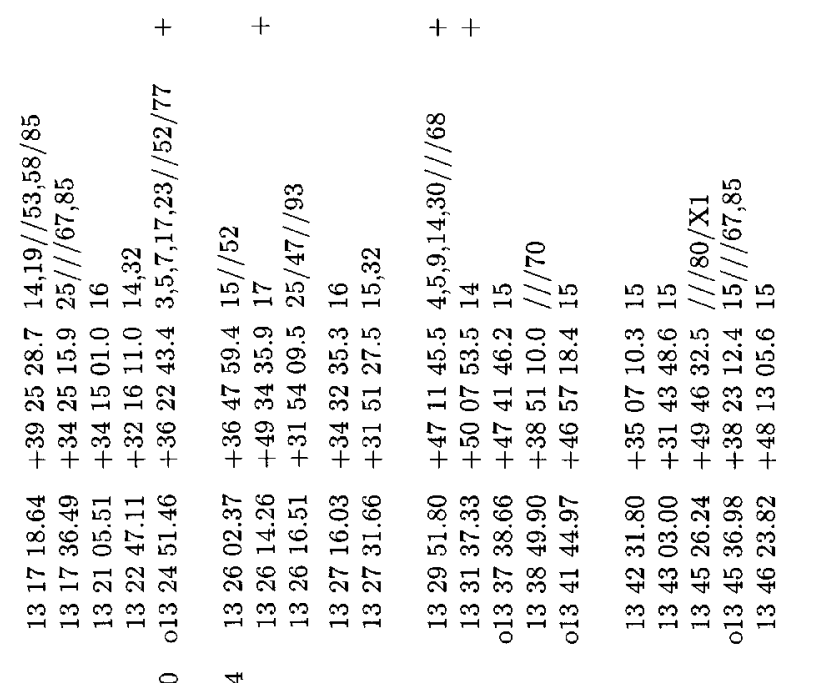

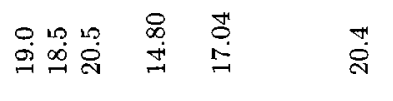

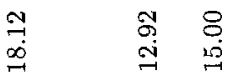

8
8

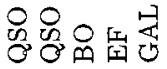

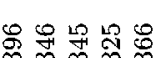

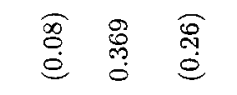

是崖岕崖

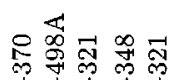

क्ञ कै

$$
\text { म }
$$

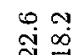

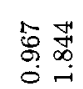

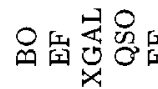

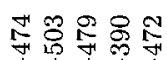

㠃冏志志总总
$++$

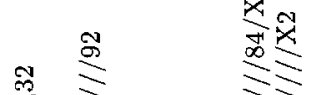

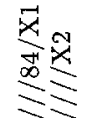

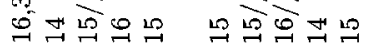

ণ⿻ $\infty$ เ

可的密完品

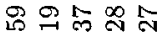

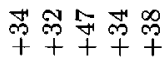

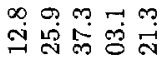

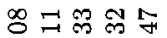

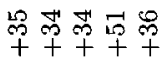

我 8

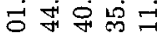

的㶽品

m

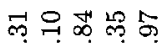

격 뼝

\%용응

寻吉吉

离空

$\underset{\sim}{\vec{N}} \stackrel{\infty}{\infty} \stackrel{\infty}{\sim}$

$\stackrel{\infty}{\infty} \stackrel{\infty}{\infty} \underset{\infty}{-}$

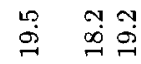

恿哭

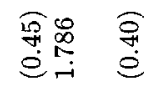

祸

兵宓完鼠

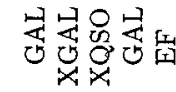

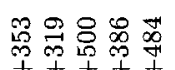

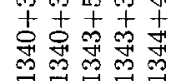

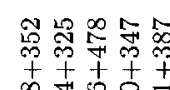

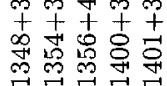

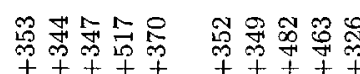

寻志志

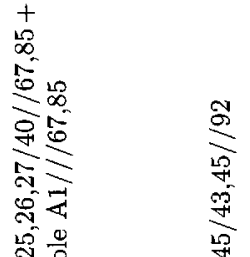

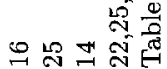

诂若

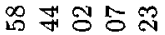

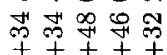

익

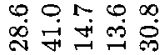
舟伿踏 节贲咕梁吊

총 $\stackrel{\infty}{\circ} \stackrel{\infty}{\circ}$

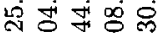

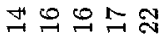

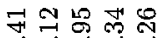

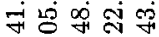

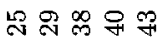
苛吉出吉

离

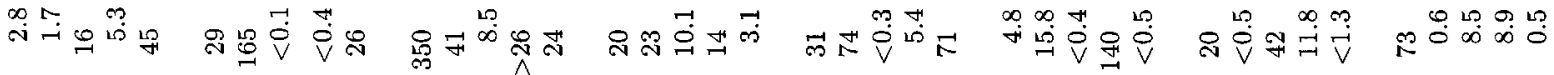

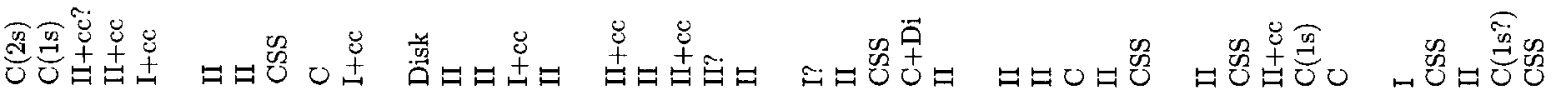
†⿺

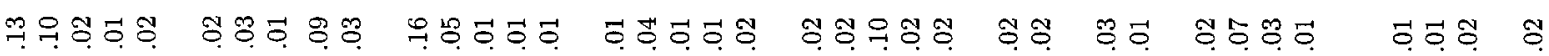

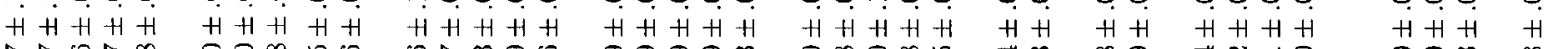

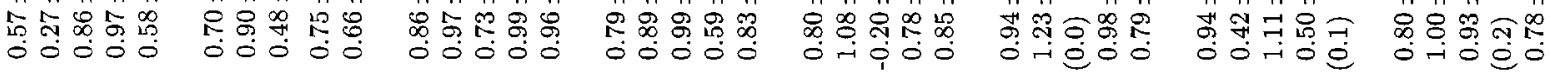

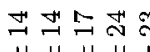

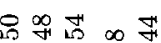

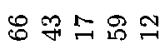

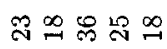

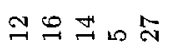

논

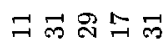

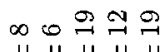

정 눙

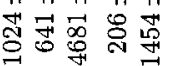

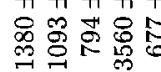

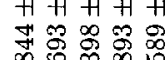

每

$H+H+H \quad H+H+H$

H + H + H H

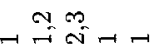
年4

$\infty$

T

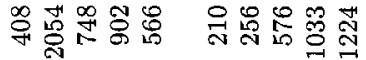

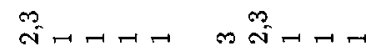
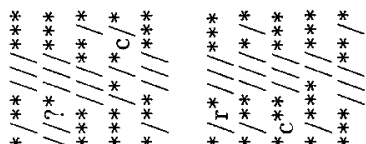

\section{옹ㅎㅀ}

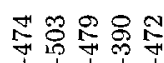

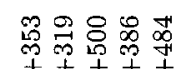

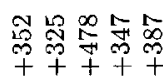

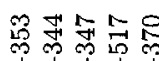

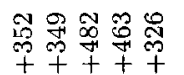

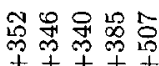
讯凩芯 
Table 3. continued

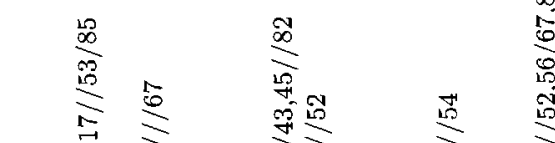
n $\infty$ o 0 O 車安家守品

뵤용요 8

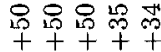

ㅇำ

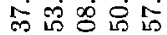

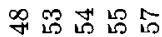

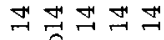
귀 우요

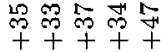

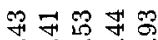

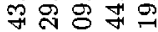
융 888
눙우웅

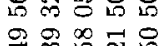

公落的年采

펑으

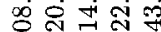

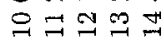

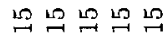

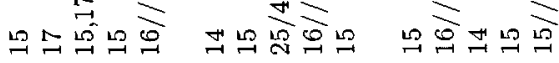

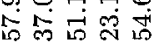

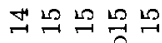

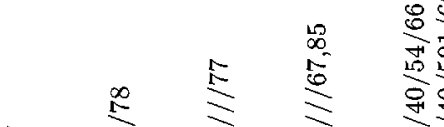

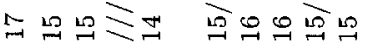
88 앵워

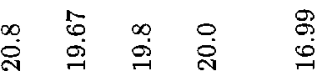

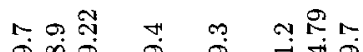

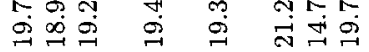

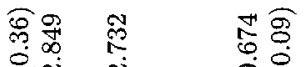

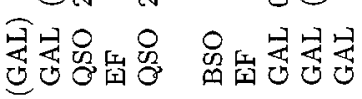

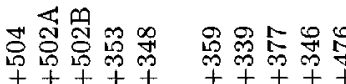

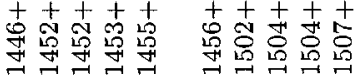
둥 요요

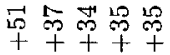

品茄令利

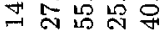
त $\stackrel{\infty}{\infty}$

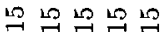

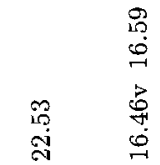

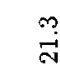

$\underset{\infty}{\infty}$

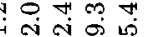

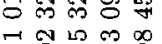

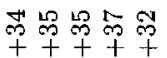

趈

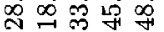

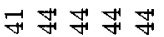

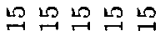

두 뚜 $\infty$

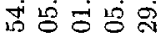

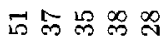

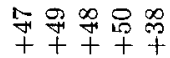

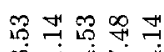

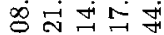

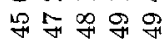

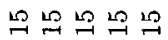

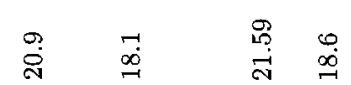

든

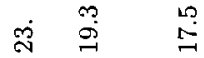

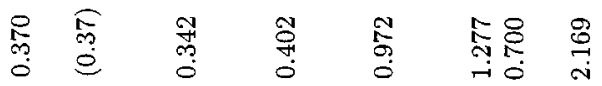

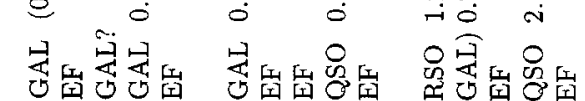

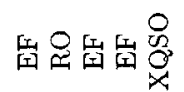

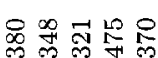

+
+
0
0
0

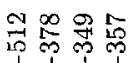

+
0
0

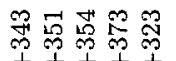

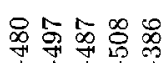

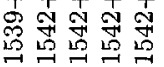

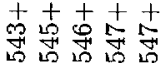

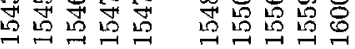

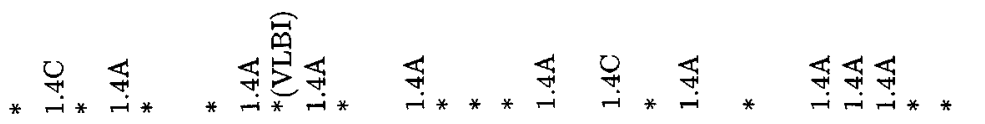

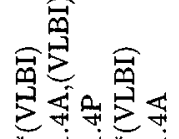

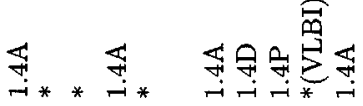

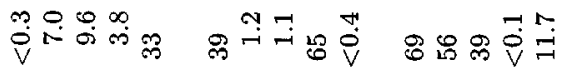

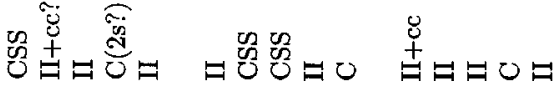

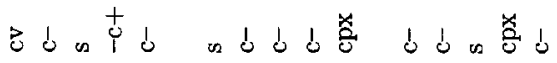
๘

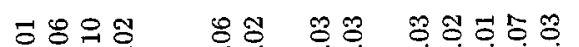

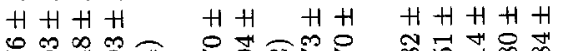

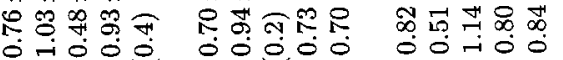

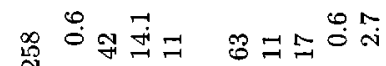

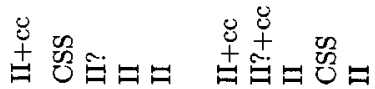
¿ d d d d d d d d d d

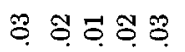
ริำ: 8 $H+H+H$

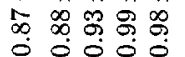

$H+H H+$ 응ㅎㅇㅇ용 $\mathrm{H}+\mathrm{H}+\mathrm{H}$ ᄋे $\&$ $\mathrm{H}+\mathrm{H}+\mathrm{H}$

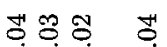

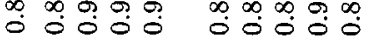

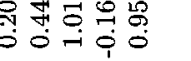

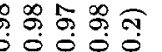
$H+H \quad H$

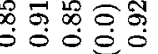

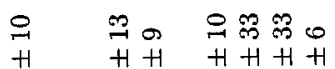
$\mathrm{H}+\mathrm{H} H \mathrm{H} H \mathrm{H} H+\mathrm{H}+\mathrm{H}$ ニニュำ

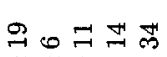
办 $\infty$ ก

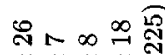

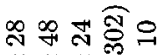

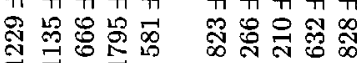
$\mathrm{H}+\mathrm{H}+\mathrm{H}+\mathrm{H}$ $H+H+1$ H H H

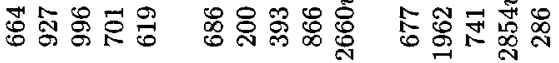

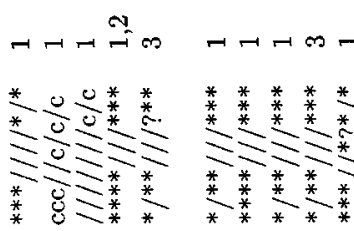
$-\stackrel{m}{i}+-1$

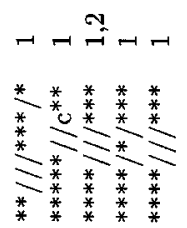
$r \stackrel{\text { s }}{n} \rightarrow-$

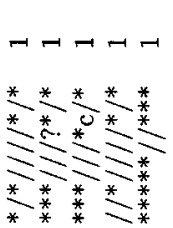
$\rightarrow \infty \stackrel{n}{n-1}$
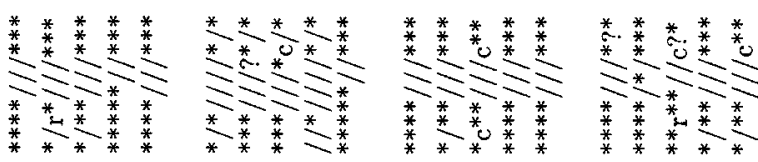
농 令骂品

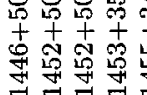

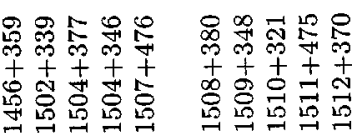

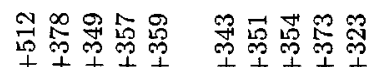

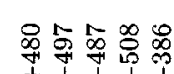

罂吉志志

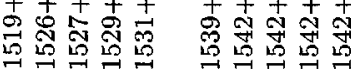

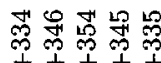

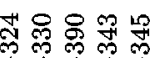

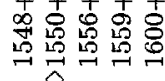


Table 3. continued

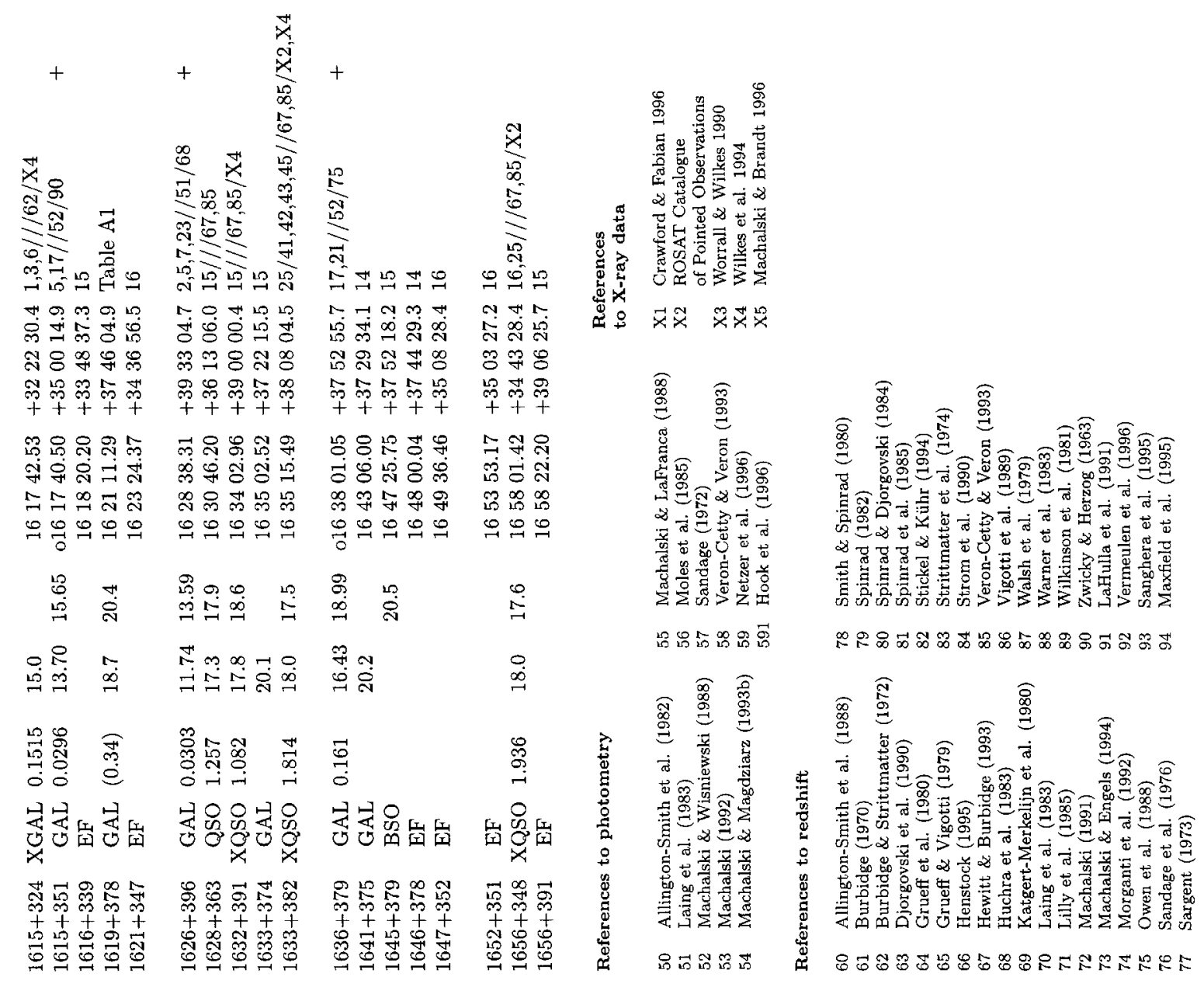

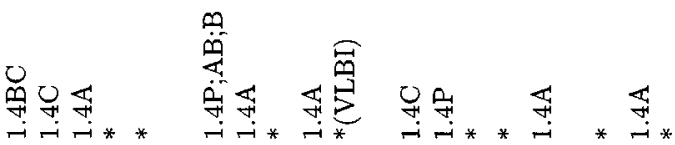

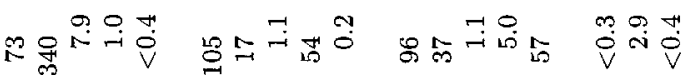

草诰语

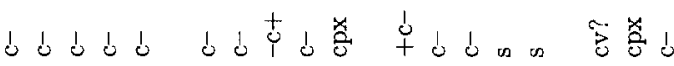

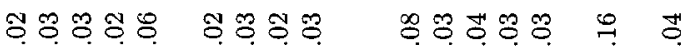

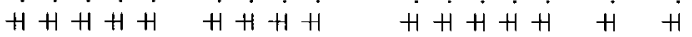

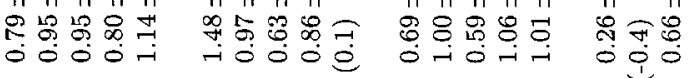

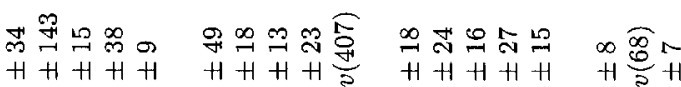

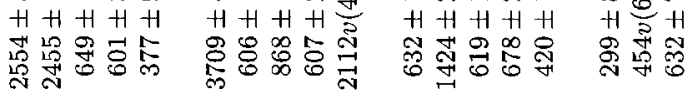

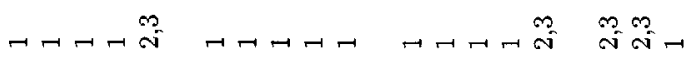

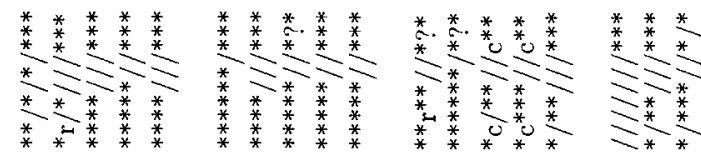

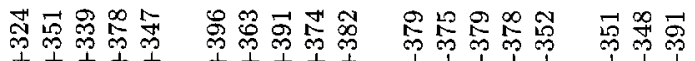

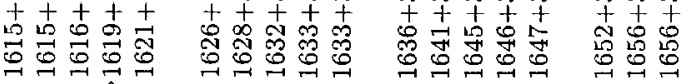

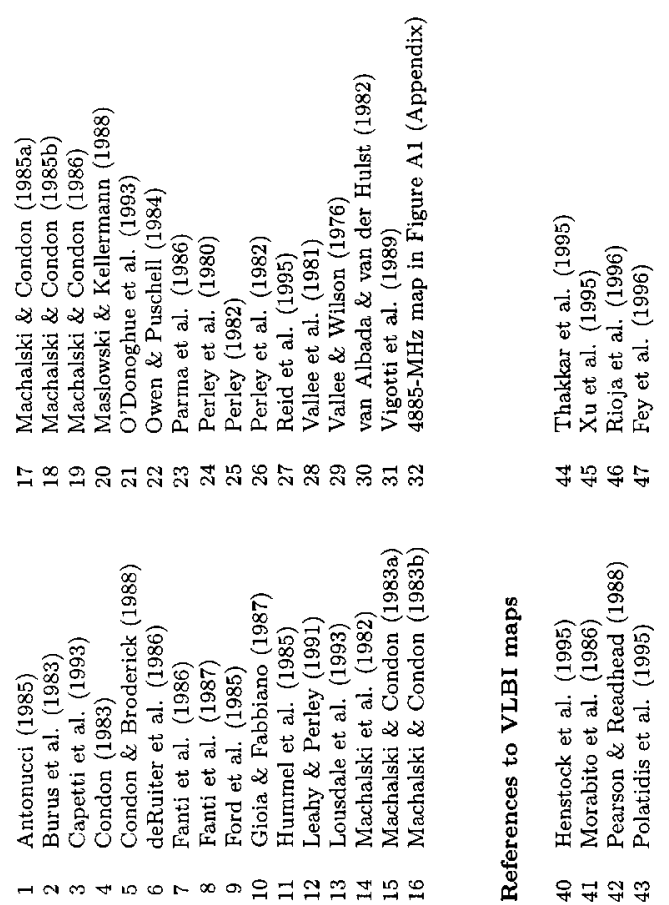

